\title{
Central Configurations and Action Minimizing Orbits in Kite Four-Body Problem
}

\author{
B. Benhammouda $\mathbb{D},{ }^{1}$ A. Mansur $\mathbb{D},{ }^{1}$ M. Shoaib $\mathbb{D}^{1},{ }^{1}$ I. Szücs-Csillik $\mathbb{D}^{2},{ }^{2}$ and D. Offin $\mathbb{D}^{3}$ \\ ${ }^{1}$ Higher Colleges of Technology, Abu Dhabi, UAE \\ ${ }^{2}$ Romanian Academy, Astronomical Institute, Cluj-Napoca, Romania \\ ${ }^{3}$ Queen's University, Mathematics and Statistics Department, Kingston, ON, Canada
}

Correspondence should be addressed to D. Offin; offind@queensu.ca

Received 19 February 2020; Accepted 22 April 2020; Published 1 August 2020

Guest Editor: Elbaz I. Abouelmagd

Copyright ( $\odot 2020$ B. Benhammouda et al. This is an open access article distributed under the Creative Commons Attribution License, which permits unrestricted use, distribution, and reproduction in any medium, provided the original work is properly cited.

\begin{abstract}
In the current article, we study the kite four-body problems with the goal of identifying global regions in the mass parameter space which admits a corresponding central configuration of the four masses. We consider two different types of symmetrical configurations. In each of the two cases, the existence of a continuous family of central configurations for positive masses is shown. We address the dynamical aspect of periodic solutions in the settings considered and show that the minimizers of the classical action functional restricted to the homographic solutions are the Keplerian elliptical solutions. Finally, we provide numerical explorations via Poincaré cross-sections, to show the existence of periodic and quasiperiodic solutions within the broader dynamical context of the four-body problem.
\end{abstract}

\section{Introduction}

To understand the dynamics presented by a total collision of the masses or the equilibrium state of a rotating system, we are led to the concept of central configurations. A configuration of $n$ bodies is central if the acceleration of each body is a scalar multiple of its position [1-4]. Let $r_{i} \in \mathbb{R}^{2}$ and $m_{i}, i=1, \ldots, n$, denote the position and the mass of the $i$ th body, respectively. Also, let $r_{i j}=\left\|r_{i}-r_{j}\right\|$ represent the distance between the $i$ th and $j$ th bodies. An $n$-body system forms a planar noncollinear central configuration $[5,6]$ if

$$
f_{i j}=\sum_{k=0, k \neq i, j}^{n-1} m_{k}\left(R_{i k}-R_{j k}\right) \Delta_{i j k}=0
$$

where $R_{i j}=r_{i j}^{-3}$ and $\Delta_{i j k}=\left(\mathbf{r}_{i}-\mathbf{r}_{j}\right) \wedge\left(\mathbf{r}_{i}-\mathbf{r}_{k}\right)$ represent the area of the triangle determined by the sides $\left\|\mathbf{r}_{i}-\mathbf{r}_{j}\right\|$ and $\left\|\mathbf{r}_{i}-\mathbf{r}_{k}\right\|$.

The four-body problem can be considered from two different perspectives. The perturbative approach where we study the dynamical aspects as a perturbation of the three- body dynamics and assume that one of the masses is vanishingly small, or the global approach where we allow the masses to vary independently and stay positive. In this paper, we take the global approach and will study analytically the problem of central configurations and their dynamical aspects.

The computation of central configurations is a difficult problem for $n \geq 4$. To overcome this difficulty, symmetries or other restriction methods are used to reduce the number of variables and obtain partial answers; see, for example, Cors and Roberts [7]; Albouy et al. [8]; Shoaib et al. [9]; Érdi and Czirják [10]. In this paper, we consider a four-body problem with one axis of symmetry so that the four different masses make a convex or concave kite.

Since the classification of central configurations as one of the problems for the 21st century by Smale [11], it has attracted a lot of attention in recent years and has helped in the understanding of the $n$-body problem [12-23]. Ji et al. [24] and Waldvogel [25] study a rhomboidal four-body problem with two pairs of masses and use Poincaré sections to find regions of stability for the rhomboidal four-body problem. In addition, 
Waldvogel [25] also takes advantage of the simplicity of the equations and study its collisions and escape manifolds. Yan [26] considers the same problem for four equal masses and studies the linear stability of its periodic orbits. One of our results will consider the same model but with only one pair of masses. We will analytically derive regions of central configurations and will also investigate the existence of periodic orbits. Mello and Fernandez [27] prove the existence of kite central configurations for four- and five-body problems on a circle. In one special case, our rhomboidal model is similar to their model for which we also identify a number of periodic orbits and discuss its action minimizing orbits. Gordon [28] has proved that the elliptic Keplerian orbit minimizes the Lagrangian action of the two-body problem with periodic boundary conditions. It is also known that the Eulerian and Lagrangian elliptical solutions for the planar three-body problem are the variational minimizers of the Lagrangian action functional $[29,30]$. In the study by Mansur and Offin [31]; Mansur et al. [32]; Mansur et al. [33], the authors have extended these ideas to prove that the homographic solutions to the constrained parallelogram four-body problem are the variational minimizers of the Lagrangian action functional. In this paper, we prove that the minimizers for the action functional restricted to the homographic solutions are the Keplerian elliptical solutions for the four-body problem with three equal and unequal masses. Perez-Chavela and Santoprete [13] show the existence of kite central configurations for a pair of symmetric masses and show that such a configuration must always possess a symmetry. Similarly, Celli [34] proves the existence of planar diamond and trapezoidal central configurations for two pairs of equal masses. Corbera and Llibre [35] give a complete classification of the same problem and show that this setup has exactly 34 different classes of central configurations.

More recently, Deng et al. [36] and Corbera et al. [37] prove that any four-body setup with perpendicular diagonals must be a kite $[35,38]$. Santoprete [39] studies a four-body problem with a pair of equal masses and a pair of parallel opposite sides and show that if the opposite masses are equal, then the four-body arrangement must have a line of symmetry and will be a kite.

The paper is organized as follows: Section 2 discusses the equations of motion for the four-body problem. Section 3 discusses the existence of central configurations and the action minimizing orbits for the four-body problem where three masses are equal and arranged at vertices of an isosceles triangle and the fourth mass is on the axis of symmetry. In Section 4, we discuss the variational techniques where the action functional corresponding to these family of solutions is shown to be a minimizer. Section 5 discusses the existence of central configurations and the action minimizing orbits for the four-body problem where two symmetric masses are equal on the horizontal axis and two nonequal masses are on the vertical axis.

\section{Equations of Motion}

Consider four positive point masses $m_{0}, m_{1}, m_{2}$, and $m_{3}$ having position vectors $\mathbf{r}_{i}$ and interbody distances $r_{i j}$. For a general four-body setup, equation (1) gives the following six central configuration equations when $n=4$ :

$$
\begin{aligned}
& f_{01}=m_{2}\left(R_{02}-R_{12}\right) \Delta_{012}+m_{3}\left(R_{03}-R_{13}\right) \Delta_{013}=0, \\
& f_{02}=m_{1}\left(R_{01}-R_{21}\right) \Delta_{021}+m_{3}\left(R_{03}-R_{23}\right) \Delta_{023}=0, \\
& f_{03}=m_{1}\left(R_{01}-R_{31}\right) \Delta_{031}+m_{2}\left(R_{02}-R_{32}\right) \Delta_{032}=0, \\
& f_{12}=m_{0}\left(R_{10}-R_{20}\right) \Delta_{120}+m_{3}\left(R_{13}-R_{23}\right) \Delta_{123}=0, \\
& f_{13}=m_{0}\left(R_{10}-R_{30}\right) \Delta_{130}+m_{2}\left(R_{12}-R_{32}\right) \Delta_{132}=0, \\
& f_{23}=m_{0}\left(R_{20}-R_{30}\right) \Delta_{230}+m_{1}\left(R_{21}-R_{31}\right) \Delta_{231}=0 .
\end{aligned}
$$

Lemma 1. Consider a four-body problem with masses $m_{0}$, $m_{1}, m_{2}$, and $m_{3}$ and position vectors $\mathbf{r}_{0}=(0, c)$, $\mathbf{r}_{1}=(-1,-a), \mathbf{r}_{2}=(0, b)$, and $\mathbf{r}_{3}=(1,-a)$, where $a>0$ and $b>c$, then

(a) The symmetric masses $m_{1}$ and $m_{3}$ are equal.

(b) The central configuration equations are

$$
\begin{aligned}
& f_{01}=m_{1}\left(R_{03}-R_{13}\right) \Delta_{013}+m_{2}\left(R_{02}-R_{12}\right) \Delta_{012}, \\
& f_{12}=m_{0}\left(R_{10}-R_{20}\right) \Delta_{120}+m_{1}\left(R_{13}-R_{23}\right) \Delta_{123} .
\end{aligned}
$$

Proof. Consider four positive masses $m_{0}, m_{1}, m_{2}$, and $m_{3}$ with position vectors $\mathbf{r}_{0}=(0, c), \mathbf{r}_{1}=(-1,-a), \mathbf{r}_{2}=(0, b)$, and $\mathbf{r}_{3}=(1,-a)$, where $a>0$ and $b>c$. Using the definitions of $R_{i j}, \Delta_{i j k}$, and $\mathbf{r}_{i}(i=0,1,2,3,4)$, we obtain

$$
\begin{aligned}
& R_{01}=R_{03}=R_{30}=\frac{1}{\left((a+c)^{2}+1\right)^{3 / 2}}, \\
& R_{13}=\frac{1}{8}, \\
& R_{02}=\frac{1}{(b-c)^{3}}, \\
& R_{12}=R_{21}=R_{32}=\frac{1}{\left((a+b)^{2}+1\right)^{3 / 2}},
\end{aligned}
$$

with

$$
\begin{aligned}
& \Delta_{i j k}=-\Delta_{j i k}=-\Delta_{i k j}=-\Delta_{k j i}, \\
& \Delta_{i j k}=\Delta_{j k i}=\Delta_{k i j}, \\
& \Delta_{i j k}=0, \quad \text { if } i=j \text { or } i=k \text { or } j=k, \\
& \Delta_{012}=\Delta_{023}=c-b, \\
& \Delta_{013}=2(a+c), \\
& \Delta_{123}=-2(a+b) .
\end{aligned}
$$

Using the symmetry of the problem and the relations (4) and (5), it is trivial to see that

$$
f_{02}=\left(m_{1}-m_{3}\right)\left(R_{01}-R_{21}\right) \Delta_{021}=0 .
$$

Since $\Delta_{021} \neq 0$ and $R_{21} \neq R_{01}$, therefore $m_{3}=m_{1}$. This completes the proof of Lemma 1 (a). 
From the geometry of the problem, $\Delta m_{1} m_{0} m_{3}$ and $\Delta m_{1} m_{2} m_{3}$ are both isosceles, and therefore $R_{01}=R_{03}$, $R_{12}=R_{32}$, and $\Delta_{032}=\Delta_{012}$ and hence $f_{03}=f_{01}$. This also implies that $f_{13} \equiv 0$. By a similar argument, it can be shown that $f_{23}=f_{12}$. This leaves two independent equations $f_{01}$ and $f_{12}$ from the set of equations given in (2). This completes the proof of Lemma 1.

\section{Three Equal Masses at the Vertices of a Triangle and a Fourth Mass on the Axis of Symmetry}

In this section, we consider a four-body problem where three equal masses $\left(m_{1}=m_{2}=m_{3}\right)$ are arranged at the vertices of an isosceles triangle and a fourth mass $m_{0}$ is on the axis of symmetry as shown in Figure 1. We start by showing the existence of central configuration for a concave kite fourbody problem and then explicitly find regions where such a configuration exists for positive masses. We also discuss the action minimizing orbits for this particular problem.

\subsection{Central Configurations}

Theorem 1. Consider four point masses $m_{0}$ and $m_{1}=m_{2}=$ $m_{3}$ having position vectors $\mathbf{r}_{0}=(0,0), \quad \mathbf{r}_{1}=(-1,-a)$, $\mathbf{r}_{2}=(0, b)$, and $\mathbf{r}_{3}=(1,-a)$, where $a>0$ and $b>0$. Then, there exists a unique mass ratio $\mu_{0}(a, b)$ :

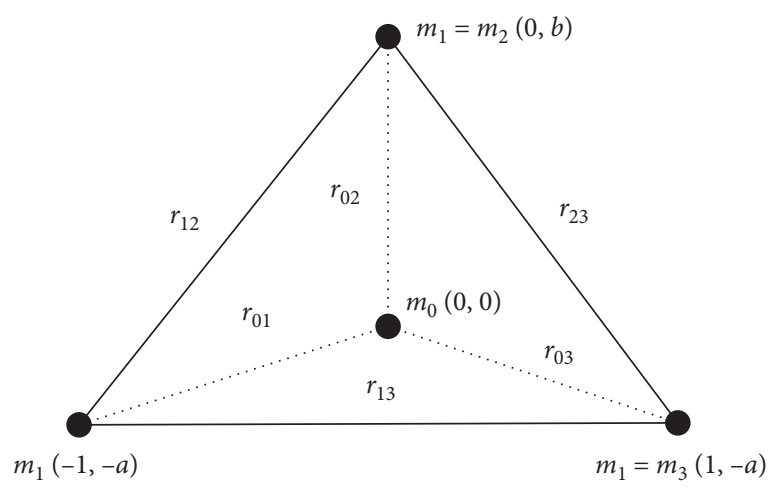

FIgURE 1: Concave kite four-body configurations with three equal masses $m_{1}=m_{2}=m_{3}$.

$$
\begin{aligned}
\mu_{0} & =\frac{m_{0}}{m_{1}} \\
& =\frac{\left(\left((a+b)^{2}+1\right)^{3 / 2}-8\right)(a+b) b^{2}\left(a^{2}+1\right)^{3 / 2}}{4\left(\left(a^{2}+1\right)^{3 / 2}-b^{3}\right)\left((a+b)^{2}+1\right)^{3 / 2}},
\end{aligned}
$$

such that $\mathbf{r}=\left(\mathbf{r}_{0}, \mathbf{r}_{1}, \mathbf{r}_{2}, \mathbf{r}_{3}\right)$ is a central configuration for $\mu_{0}>0$ in $R_{\mu_{0}}(a, b)$ subject to the constraint $g(a, b)=0$. The region $R_{\mu_{0}}(a, b)$ and the constraint $g(a, b)$ are given below:

$$
\begin{aligned}
R_{\mu_{0}}(a, b) & =\left\{(a, b) \mid\left(\frac{2}{\sqrt{3}}<b<\sqrt{3} \wedge\left(0<a<\sqrt{3}-b \vee a>\sqrt{b^{2}-1}\right)\right) \vee\left(b \geq \sqrt{3} \wedge a>\sqrt{b^{2}-1}\right)\right\}, \\
g(a, b) & =-b\left(\frac{1}{b^{3}}-\frac{1}{\alpha}\right)+2 a\left(-\frac{1}{8}+\frac{1}{\beta}\right)=0
\end{aligned}
$$

where $\alpha=\left((a+b)^{2}+1\right)^{3 / 2}$ and $\beta=\left(a^{2}+1\right)^{3 / 2}$.

Before we attempt to prove Theorem 1, we will need help from the following lemmas.

Lemma 2. The function $g$ defined by (8) is negative for all $0<a<\sqrt{3}$ and $0<b \leq B=(2 / \sqrt{3})((3 / 7)+(2 \sqrt{3} / 7))^{1 / 4} \simeq$ 1.13 .

Proof. Let $0<a<\sqrt{3}$ and $0<b \leq B$, then we have

$$
\frac{g(a, b)}{b}=\frac{1}{\alpha}+\frac{2 a}{b}\left(\frac{1}{\beta}\right)-\frac{4+a b^{2}}{4 b^{3}} \text {. }
$$

Consider the equation of the straight line segment that lies in the first quadrant:

$$
x+\frac{2 a}{b} y-\frac{4+a b^{2}}{4 b^{3}}=0 .
$$

For positive $x$ and $y$,

$$
x+\frac{2 a}{b} y-\frac{4+a b^{2}}{4 b^{3}}<0
$$

is equivalent to

$$
\begin{aligned}
& x<\frac{4+a b^{2}}{4 b^{3}}, \\
& y<\frac{4+a b^{2}}{8 a b^{2}} .
\end{aligned}
$$

To prove that $g(a, b) b^{-1}<0$, we need to show that

$$
\begin{aligned}
& \frac{1}{\alpha}<\frac{4+a b^{2}}{4 b^{3}}, \\
& \frac{1}{\beta}<\frac{4+a b^{2}}{8 a b^{2}} .
\end{aligned}
$$

For $(1 / \alpha)<\left(4+a b^{2} / 4 b^{3}\right)$, it is trivial to see that

$$
\frac{1}{\alpha}=\frac{1}{\left(1+(a+b)^{2}\right)^{3 / 2}}<\frac{1}{b^{3}}<\frac{4+a b^{2}}{4 b^{3}} .
$$

Similarly, to show that $(1 / \beta)<\left(4+a b^{2} / 8 a b^{2}\right)$, we need 


$$
\begin{gathered}
\beta>\frac{8 a b^{2}}{4+a b^{2}}, \\
\text { or }\left(1+a^{2}\right)^{3}>\left(\frac{8 a b^{2}}{4+a b^{2}}\right)^{2} .
\end{gathered}
$$

Inequality (16) is equivalent to

$$
\left(1+a^{2}\right)^{3}\left(4+a b^{2}\right)^{2}-64 a^{2} b^{4}>0 .
$$

Expanding the left-hand side of (17), we obtain the following:

$$
\begin{aligned}
p(a, b)= & b^{4} a^{8}+8 b^{2} a^{7}+\left(16+3 b^{4}\right) a^{6}+24 b^{2} a^{5} \\
& +\left(48+3 b^{4}\right) a^{4}+24 b^{2} a^{3}+\left(48-63 b^{4}\right) a^{2}+8 b^{2} a+16
\end{aligned}
$$

For positive $a$ and $b$,

$$
\begin{aligned}
p(a, b) & >48 a^{4}+\left(48-63 b^{4}\right) a^{2}+16 \\
& \geq 48 a^{4}+\left(48-63 B^{4}\right) a^{2}+16 \\
& =48\left(a^{2}-\frac{1}{\sqrt{3}}\right)^{2} \geq 0 .
\end{aligned}
$$

Consequently, $g(a, b)<0$ for $0<a<\sqrt{3}$ and $0<b \leq B$. This completes the proof of Lemma 2 .

Lemma 3. The partial derivative of $g$ defined by (3) satisfies $g_{b}(a, b)>\left(3 / \alpha^{2}\right)$, for all $a>0$ and $b>0$.

Proof. For positive $a$ and $b$, we have

$$
\begin{aligned}
g_{b}(a, b) & =\frac{2}{b^{3}}+\frac{\alpha-b \alpha_{b}}{\alpha^{2}}, \\
& =\frac{2}{b^{3}}+\frac{\alpha-3 b(a+b) \gamma}{\alpha^{2}},
\end{aligned}
$$

where $\gamma=\left(1+(a+b)^{2}\right)^{1 / 2}$ and $\alpha_{b}$ is the derivative of $\alpha$ w.r.t. $b$.

Since $b^{3}<\alpha$ for all $a>0$ and $b>0$, then

$$
g_{b}(a, b)>\frac{2}{\alpha}+\frac{\alpha-3 b(a+b) \gamma}{\alpha^{2}} \text {. }
$$

Using the fact that $\alpha=\gamma^{3}$ and $\gamma^{2}=1+(a+b)^{2}$, we get

$$
g_{b}(a, b)>\frac{3 \sqrt{1+(a+b)^{2}}\left(1+a^{2}+a b\right)}{\gamma^{6}} .
$$

Since $a$ and $b$ are positive, therefore

$$
g_{b}(a, b)>\frac{3}{\alpha^{2}}
$$

This completes the proof of Lemma 3.

Remark 1. Numerically, it is easy to show that $g(a, 2)=0$ when $a=0.14$ and $a=1.32$. For $a \in I=(0.14,1.32)$, $g(a, 1)<0$ and $g(a, 2)>0$, when $a \in I$, see Figure 2 .

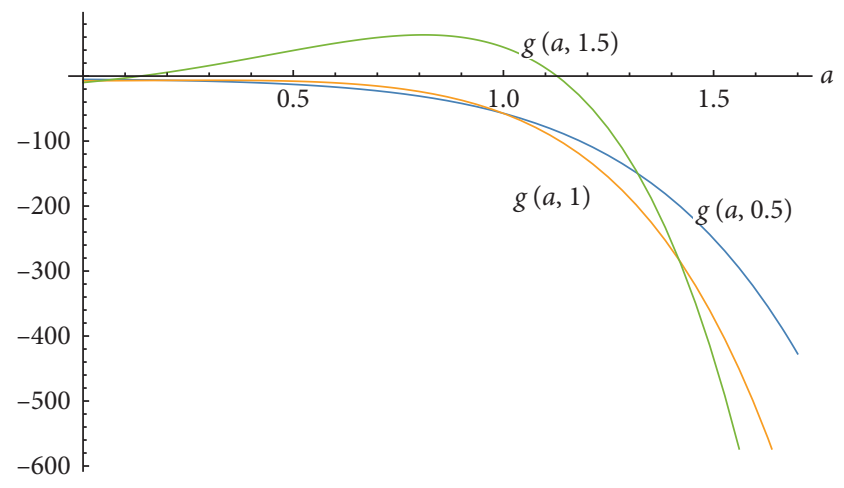

Figure 2: Graph of $g(a, b)$ for values of $b=0.5,1$, and 1.5.

Therefore, by intermediate value theorem $g(a, b)$ has at least one root $b \in(1,2)$ when $a \in I$.

Lemma 4. Consider the function $g$ defined by (3). Then, for any $a_{0} \in I=(0.14,1.32)$ there exists an interval $U \subset I$ containing $a_{0}$ and an interval $V \subset(1,2)$ containing $b_{0}$ such that there is a unique continuously differentiable function $b=$ $\psi(a)$ defined on $U$ with $b \in V$ that satisfies $g(a, b)=0$.

Proof. Let $a_{0}$ be any number in the interval $I=(0.14,1.32)$; then, using Lemma 3 , we have $g\left(a_{0}, 1\right)<0$. Then, numerically, one can check that $g\left(a_{0}, 2\right)>0$ for $a_{0} \in I$. Thus, by the mean value theorem, there exists at least one $b_{0} \in(1,2)$, solution of $g\left(a_{0}, b\right)=0$. By Lemma $3, g_{b}\left(a_{0}, b\right)>0$ for all $b>0$, hence the solution $b_{0}$ is unique. Since $g$ has continuous partial derivatives and $g\left(a_{0}, b_{0}\right)=0$, with $g_{b}\left(a_{0}, b_{0}\right) \neq 0$, then by the implicit function theorem, there exists an open interval $U \subset I$ containing $a_{0}$ and an interval $V \subset(1,2)$ containing $b_{0}$ such that there is a unique continuously differentiable function $b=\psi(a)$ defined on $U$ with $b \in V$ that satisfies $g(a, b)=0$. This completes the proof of Lemma 4.

Proof of Theorem 1. Let $m_{1}=m_{2}=m_{3}$ and $c=0$, and then from Lemma 1 , we obtain the following central configuration equations:

$$
\begin{aligned}
& f_{01}=\left(R_{01}-R_{13}\right) \Delta_{013}+\left(R_{02}-R_{12}\right) \Delta_{012}=0, \\
& f_{12}=m_{0}\left(R_{01}-R_{02}\right) \Delta_{012}+m_{1}\left(R_{13}-R_{12}\right) \Delta_{123}=0 .
\end{aligned}
$$

Solving the above equations, we obtain

$$
\mu_{0}(a, b)=\frac{m_{0}}{m_{1}}=\frac{\phi_{1}(a, b)(a+b) b^{2}\left(a^{2}+1\right)^{3 / 2}}{4 \phi_{2}(a, b)\left((a+b)^{2}+1\right)^{3 / 2}}
$$

such that constraint (3) holds, where $\phi_{1}(a, b)=\left((a+b)^{2}+1\right)^{3 / 2}-8$ and $\phi_{2}(a, b)=\left(a^{2}+1\right)^{3 / 2}-$ $b^{3}$. It is proved in Lemmas 2,3 , and 4 that constraint (8) is satisfied by showing the existence of a smooth curve:

$$
\psi(a)=\{(a, b) \mid g(a, b)=0,0<a<\sqrt{3}, 1<b<\sqrt{2}\} .
$$

To find the region where $\mu_{0}>0$, we solve the following inequality for $a$ and $b$ : 


$$
\frac{\phi_{1}(a, b)}{\phi_{2}(a, b)}>0 .
$$

The functions $\phi_{1}(a, b)$ and $\phi_{2}(a, b)$ are positive in $R_{\phi_{1}}$ and $R_{\phi_{2}}$, respectively, where

$$
\begin{aligned}
& R_{\phi_{1}}=\{(a, b) \mid(0<b \leq \sqrt{3} \wedge a>\sqrt{3}-b) \vee(b>\sqrt{3} \wedge a>0)\}, \\
& R_{\phi_{2}}=\left\{(a, b) \mid(0<b \leq 1 \wedge a>0) \vee\left(b>1 \wedge a>\sqrt{b^{2}-1}\right)\right\} .
\end{aligned}
$$

Therefore, the configuration $\mathbf{r}=\left(\mathbf{r}_{0}, \mathbf{r}_{1}, \mathbf{r}_{2}, \mathbf{r}_{3}\right)$ shown in Figure 1 forms a central configuration in $R_{\mu_{0}}=\left(R_{\phi_{1}} \cap R_{\phi_{2}}\right) \cup\left(R_{\phi_{1}}^{c} \cap R_{\phi_{2}}^{c}\right)$, where

$$
\begin{aligned}
R_{\mu_{0}}= & \left(R_{\phi_{1}} \cap R_{\phi_{2}}\right) \cup\left(R_{\phi_{1}}^{c} \cap R_{\phi_{2}}^{c}\right) \\
= & \left\{(a, b) \mid(0<b \leq 1 \wedge a>\sqrt{3}-b) \vee\left(1<b<\frac{2}{\sqrt{3}} \wedge\left(0<a<\sqrt{b^{2}-1} \vee(a>\sqrt{3}-b)\right)\right)\right. \\
& \left.\vee\left(b=\frac{2}{\sqrt{3}} \wedge\left(0<a<\frac{1}{\sqrt{3}} \vee\left(a>\frac{1}{\sqrt{3}}\right)\right)\right) \vee\left(\frac{2}{\sqrt{3}}<b<\sqrt{3} \wedge\left(0<a<\sqrt{3}-b \vee a>\sqrt{b^{2}-1}\right)\right) \vee\left(b \geq \sqrt{3} \wedge a>\sqrt{b^{2}-1}\right)\right\},
\end{aligned}
$$

such that (8) holds. Since $g(a, b)=0$ has an absolute minimum at $\left(a_{0}, b_{0}\right)=(0.53,1.15)$ and is monotonically decreasing for $0<a<0.53$ and increasing for $a>0.53$.
Therefore, $g(a, b) \neq 0$ for $b<1.15$. Hence, the region $R_{\mu_{0}}$ simplifies to

$$
R_{\mu_{0}}=\left\{(a, b) \mid\left(\frac{2}{\sqrt{3}}<b<\sqrt{3} \wedge\left(0<a<\sqrt{3}-b \vee a>\sqrt{b^{2}-1}\right) \vee\left(b \geq \sqrt{3} \wedge a>\sqrt{b^{2}-1}\right)\right)\right\} .
$$

The region $R_{\mu_{0}}$ is shown in Figure 3. This completes the proof of Theorem 1.

To be able to comment on the values of $\mu_{0}$ in the central configuration region, we use interpolation techniques and write the solution of $g(a, b)=0$ as $b=\psi(a)$, where

$$
\begin{aligned}
\psi(a)= & -99.4142 a^{11}+713.882 a^{10}-2296.59 a^{9} \\
& +4371.32 a^{8}-5475.35 a^{7}+4748.21 a^{6} \\
& -2919.84 a^{5}+1282.71 a^{4}-400.891 a^{3} \\
& +88.7673 a^{2}-13.8585 a+2.4259 .
\end{aligned}
$$

The function $\psi(a)$ accurately approximates the solution of $g(a, b)=0$ in the central configuration region where $\mu_{0}$ is positive. The approximation error is between $10^{-10}$ and $10^{-6}$. This gives $\mu_{0}$ as a function of $a$ as follows:

$$
\mu_{0}(a)=\frac{\left.\left(\left((a+\psi(a))^{2}+1\right)\right)^{3 / 2}-8\right)(a+\psi(a)) \psi^{2}(a)\left(a^{2}+1\right)^{3 / 2}}{4\left(\left(a^{2}+1\right)^{3 / 2}-\psi(a)^{3}\right)\left((a+\psi(a))^{2}+1\right)^{3 / 2}} .
$$

The function $\mu_{0}(a)$ is a bounded, well-defined continuous function of $a$ except when $q(a)=a^{2}+1-\psi(a)^{2}=0$. To identify the values of $a$ where $q(a)=0$, we write it as

$$
\begin{aligned}
q(a)= & -9883.18 a^{22}+141940 . a^{21}-966255 . a^{20} \\
& +4.14813 \times 10^{6} a^{19}-1.26042 \times 10^{7} a^{18} \\
& +2.88398 \times 10^{7} a^{17}-5.16176 \times 10^{7} a^{16} \\
& +7.41023 \times 10^{7} a^{15}-8.68138 \times 10^{7} a^{14} \\
& +8.40051 \times 10^{7} a^{13}-6.77049 \times 10^{7} a^{12} \\
& +4.57074 \times 10^{7} a^{11}-2.59398 \times 10^{7} a^{10} \\
& +1.2402 \times 10^{7} a^{9}-5.00236 \times 10^{6} a^{8} \\
& +1.705 \times 10^{6} a^{7}-492405 . a^{6}+120891 . a^{5} \\
& -25214.6 a^{4}+4405.41 a^{3}-621.741 a^{2} \\
& +67.2389 a-4.88501=0 .
\end{aligned}
$$

The numerical solution of $q(a)=0$ shows that it has three real roots $a=1.14605, a=1.2471$, and $a=1.44556$. However, a careful observation of the region of existence of central configuration for the four-body problem in Figure 1 shows that $a=1.14605$ defines a boundary between the region of existence and nonexistence and $a=1.2471$ and $a=$ 1.44556 are outside the domain of interest. Hence, $\mu_{0}(a)$ uniquely defines the positive values of the mass ratio $m_{0} / m_{3}$ for the four-body problem as described in Theorem 1 . The region of existence of central configuration for the four-body problem with three equal masses is given in Figure 3. Taking 


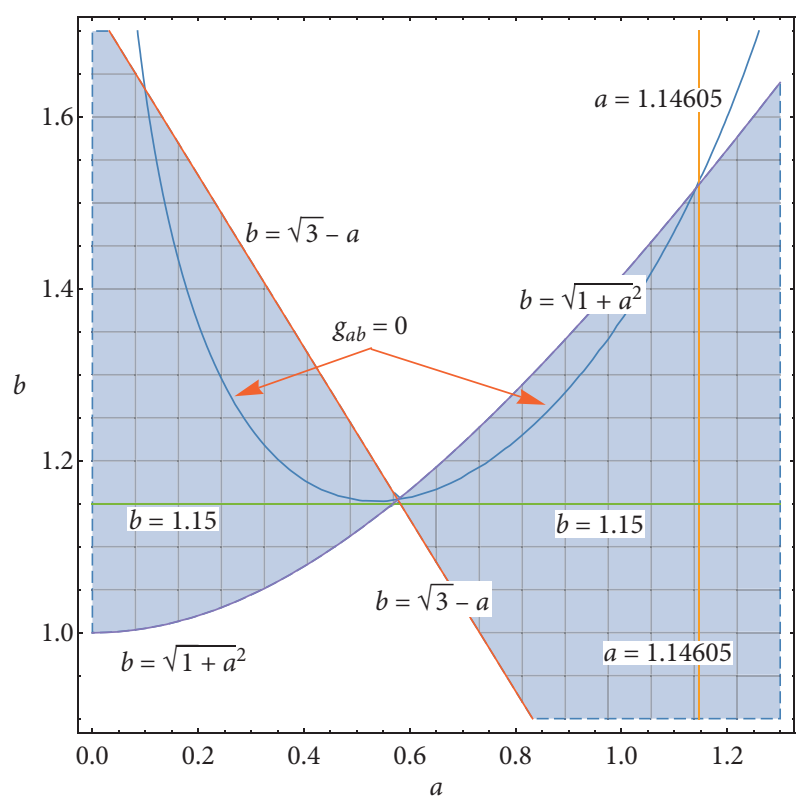

FIgURE 3: Region of existence of central configuration for the concave kite four-body problem with $\left(m_{1}=m_{2}=m_{3}, m_{0}\right)$.

advantage of the interpolated expression $b=\psi(a), \mu_{0}(a)$ is given for $b \in(0.1,1.14)$ in Figure 4 which shows $\mu_{0}(a)$ to be an increasing function of $a$ with the minimum and maximum at the end points of the domain.

3.2. Action Minimizing Orbits in the Triangular Four-Body Problem. In this section, we introduce the analytical description of a family of periodic solutions in the four-body problem using variational techniques.

Theorem 2. For the four-body problem considered in Theorem 1, the minimizers for $A(q)$ restricted to the homographic solutions $q_{i}(t)=\phi(t) q_{i, 0}$ are precisely the Keplerian elliptical solutions and the minimum of the action is equal to $(3 / 2)(2 \pi)^{2 / 3} T^{1 / 3}(\xi(a, b) / \eta(a, b))^{2 / 3}$.

Define the Lagrangian action as

$$
A(q)=\int_{0}^{T} L(q(t), \dot{q}(t)) \mathrm{d} t,
$$

where the Lagrangian $L$ is defined by

$$
\begin{aligned}
L(q, \dot{q}) & =\sum_{i=1}^{4} \frac{m_{i}}{2}\|\dot{q}\|^{2}-U(q), \\
U(q) & =\sum_{i<j} \frac{m_{i} m_{j}}{\left\|q_{i}-q_{j}\right\|} .
\end{aligned}
$$

Let us call $\Omega_{c m}$ the $y$-coordinate of the center of mass in the configuration described earlier in Section 2, then

$$
\Omega_{c m}=\frac{(b-2 a) m_{1}}{3 m_{1}+m_{0}}
$$

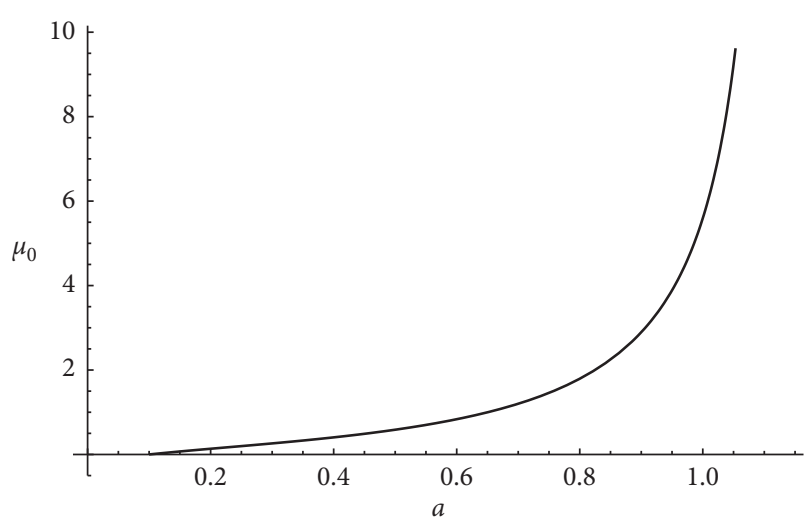

FIgURE 4: Values of $\mu_{0}(a)$ in the region of central configuration.

and the center of mass is

$$
\operatorname{COM}=\left(0, \Omega_{c m}\right) .
$$

In this case, we have the following Cartesian coordinates for the points $q_{0,0}, q_{1,0}, q_{2,0}, q_{3,0}$ :

$$
\begin{aligned}
& q_{0,0}=\left(0, \Omega_{c m}\right), \\
& q_{1,0}=\left(-1,-a-\Omega_{c m}\right), \\
& q_{2,0}=\left(0, b-\Omega_{c m}\right), \\
& q_{3,0}=\left(1,-a-\Omega_{c m}\right) .
\end{aligned}
$$

Observe that

$$
\begin{aligned}
& r_{0}=\left|q_{0,0}\right|=\Omega_{c m}, \\
& r_{1}=r_{3}=\sqrt{1+\left(a+\Omega_{c m}\right)^{2}}, \\
& r_{2}=b-\Omega_{c m} .
\end{aligned}
$$

We focus on solutions of the form $q_{i}(t)=\phi(t) q_{i, 0}$. These solutions are called homographic solutions. We will restrict the action functional to solutions of this type.

Proof of Theorem 2. The kinetic energy term $K$ is equal to

$$
\begin{aligned}
K & =\frac{1}{2} \sum_{i=0}^{3} m_{i}\left|\dot{q}_{i}(t)\right|^{2}=|\dot{\phi}(t)|^{2} r_{1}^{2}\left(m_{1}+\frac{1}{2}\left(m_{0}\left(\frac{r_{0}}{r_{1}}\right)^{2}+m_{1}\left(\frac{r_{2}}{r_{1}}\right)^{2}\right)\right) \\
& =\left|\dot{q}_{i}(t)\right|^{2}\left(m_{1}+\frac{1}{2}\left(m_{0}\left(\frac{r_{0}}{r_{1}}\right)^{2}+m_{1}\left(\frac{r_{2}}{r_{1}}\right)^{2}\right)\right),
\end{aligned}
$$

where we have used $\left|\dot{q}_{1}(t)\right|^{2}=|\dot{\phi}(t)|^{2} r_{1}^{2}$. The potential energy is given by

$$
U=\sum_{0 \leq i<j \leq 3} \frac{m_{i} m_{j}}{\left|q_{i}-q_{j}\right|}
$$

and using $\left|q_{i}-q_{j}\right|=|\phi(t)|\left|q_{i, 0}-q_{j, 0}\right|$, we get 


$$
\begin{aligned}
U= & \frac{m_{0} m_{1}}{|\phi(t)|}\left(\frac{2}{\sqrt{a^{2}+1}}+\frac{1}{b}\right) \\
& +\frac{m_{1}^{2}}{|\phi(t)|}\left(\frac{2}{\sqrt{(a+b)^{2}+1}}+\frac{1}{2}\right) .
\end{aligned}
$$

Multiplying and dividing by $r_{1}$, we obtain

$$
\begin{aligned}
U= & \frac{1}{\left|q_{1}\right|}\left(m_{0} m_{1} r_{1}\left(\frac{2}{\sqrt{a^{2}+1}}+\frac{1}{b}\right)\right. \\
& \left.+m_{1}^{2} r_{1}\left(\frac{2}{\sqrt{(a+b)^{2}+1}}+\frac{1}{2}\right)\right) .
\end{aligned}
$$

As defined previously, we use $\left(m_{0} / m_{1}\right)=\mu_{0}$, and by letting $m_{1}=1$, we have

$$
\begin{aligned}
U= & \frac{1}{\left|q_{1}\right|}\left(\mu_{0} r_{1}\left(\frac{2}{\sqrt{a^{2}+1}}+\frac{1}{b}\right)\right. \\
& \left.+r_{1}\left(\frac{2}{\sqrt{(a+b)^{2}+1}}+\frac{1}{2}\right)\right) .
\end{aligned}
$$

Now, we are ready to compute the action restricted to this class of homographic solutions. We have

$$
\begin{aligned}
A= & \int_{0}^{T}\left(1+\frac{1}{2}\left(m_{0}\left(\frac{r_{0}}{r_{1}}\right)^{2}+\left(\frac{r_{2}}{r_{1}}\right)^{2}\right)\right) \frac{\left|\dot{q}_{1}\right|^{2}}{2} \mathrm{~d} t \\
& +\int_{0}^{T}\left(\mu_{0} r_{1}\left(\frac{2}{\sqrt{a^{2}+1}}+\frac{1}{b}\right)+r_{1}\left(\frac{2}{\sqrt{(a+b)^{2}+1}}+\frac{1}{2}\right)\right) \frac{1}{\left|q_{1}\right|} \mathrm{d} t \\
= & \left(2+\left(m_{0}\left(\frac{r_{0}}{r_{1}}\right)^{2}+\left(\frac{r_{2}}{r_{1}}\right)^{2}\right)\right) \int_{0}^{T} \frac{\left|\dot{q}_{1}\right|^{2}}{2} \mathrm{~d} t \\
& +\left(\mu_{0} r_{1}\left(\frac{2}{\sqrt{a^{2}+1}}+\frac{1}{b}\right)+r_{1}\left(\frac{2}{\sqrt{(a+b)^{2}+1}}+\frac{1}{2}\right)\right) \int_{0}^{T} \frac{1}{\left|q_{1}\right|} \mathrm{d} t .
\end{aligned}
$$

Let

$$
\begin{aligned}
\eta(a, b)= & 2+\left(m_{0}\left(\frac{r_{0}}{r_{1}}\right)^{2}+\left(\frac{r_{2}}{r_{1}}\right)^{2}\right) \\
\xi(a, b)= & \mu_{0} r_{1}\left(\frac{2}{\sqrt{a^{2}+1}}+\frac{1}{b}\right) \\
& +r_{1}\left(\frac{2}{\sqrt{(a+b)^{2}+1}}+\frac{1}{2}\right) .
\end{aligned}
$$

Then,

$$
A(q)=\eta \int_{0}^{T} \frac{\left|\dot{q}_{1}\right|^{2}}{2} \mathrm{~d} t+\xi \int_{0}^{T} \frac{1}{\left|q_{1}\right|} \mathrm{d} t
$$

The infimum of $A(q)$ is

$$
\begin{aligned}
\inf _{q} A(q) & =\inf _{a>0, b>0} \inf _{q_{1}}\left\{\eta \int_{0}^{T} \frac{\left|\dot{q}_{1}\right|^{2}}{2} \mathrm{~d} t+\xi \int_{0}^{T} \frac{1}{\left|q_{1}\right|} \mathrm{d} t\right\} \\
& =\inf _{a>0, b>0}\left\{\eta \inf _{q_{1}}\left(\int_{0}^{T} \frac{\left|\dot{q}_{1}\right|^{2}}{2} \mathrm{dt}+\frac{\xi}{\eta} \int_{0}^{T} \frac{1}{\left|q_{1}\right|} \mathrm{d} t\right)\right\}
\end{aligned}
$$

By Gordon's result, we have

$$
\inf _{q_{1}}\left(\int_{0}^{T} \frac{\left|\dot{q}_{1}\right|^{2}}{2} \mathrm{~d} t+\frac{\xi}{\eta} \int_{0}^{T} \frac{1}{\left|q_{1}\right|} \mathrm{d} t\right)=\frac{3}{2}(2 \pi)^{2 / 3} T^{1 / 3}\left(\frac{\xi(a, b)}{\eta(a, b)}\right)^{2 / 3} .
$$

Then,

$$
\begin{aligned}
\inf _{q} A(q) & =\inf _{a>0, b>0}\left\{\eta(a, b) \frac{3}{2}(2 \pi)^{2 / 3} T^{1 / 3}\left(\frac{\xi(b, c)}{\eta(b, c)}\right)^{2 / 3}\right\} \\
& =\inf _{a>0, b>0}\left\{\frac{3}{2}(2 \pi)^{2 / 3} T^{1 / 3} \eta(a, b)^{1 / 3}(\xi(a, b))^{2 / 3}\right\} .
\end{aligned}
$$

Let $\phi(a, b)=(3 / 2)(2 \pi)^{2 / 3} T^{1 / 3} \eta(a, b)^{1 / 3}(\xi(a, b))^{2 / 3}$, then $\phi(a, b)$ attains its infimum at $\left(a_{0}, b_{0}\right)$ if and only if $\eta(a, b)(\xi(a, b))^{2}$ attains its infimum at $\left(a_{0}, b_{0}\right)$. It is challenging to see that for positive values of $a_{0}$ and $b_{0}$, the function $\phi(a, b)$ is convex and coercive. However, we have proved that $b$ can be written as $b=\psi(a)$ and used interpolation to find $\psi(a)$ as given in equation (31). Hence, we can now write $\eta(a, b), \xi(a, b)$, and $\phi(a, b)$ as functions of one variable. For convexity, we use the second derivative test and show that $\left(\partial^{2} \phi(a) / \partial a^{2}\right)>0$ when $a \in(0.1,1.14)$ Hence, $\phi(a)$ is convex when $a \in(0.1,1.14)$. For coercivity, we see that $\phi(a)$ is continuous for all positive values of $a$, $\phi(a) \longrightarrow \infty$ as $a \longrightarrow 0$, and when $a \longrightarrow \infty, \phi(a) \longrightarrow \infty$, which implies $\phi(a)$ is coercive. Hence, $\phi(a)$ attains $\inf _{a>0}\{\phi(a)\}$ at unique $a_{0}>0$ and satisfies $\phi\left(a_{0}\right)=0$.

\section{Three Unequal Masses $m_{3}=m_{1} \neq m_{2} \neq m_{0}$}

In this section, we discuss a four-body problem which has two symmetric equal masses $\left(m_{3}=m_{1}\right)$ on the horizontal axis and two nonequal masses $m_{0}$ and $m_{2}$ on the vertical axis. The position vectors of the four masses $m_{0}, m_{1}, m_{2}$, and $m_{3}$ are $\mathbf{r}_{0}=(0, c), \mathbf{r}_{1}=(-1,0), \mathbf{r}_{2}=(0, b)$, and $\mathbf{r}_{3}=(1,0)$, respectively. For $c>0$, the four masses make an isosceles triangle with $m_{0}$ inside the triangle (Figure 5), and for $c<0$, the convex kite configuration is obtained (Figure 6).

\subsection{Central Configurations}

Theorem 3. Consider four point masses $m_{0}, m_{1}=m_{3} \neq m_{2}$ having position vectors $\mathbf{r}_{0}=(0, c), \mathbf{r}_{1}=(-1,0), \mathbf{r}_{2}=(0, b)$, and $\mathbf{r}_{3}=(1,0)$, where $b>0$ and $b>c$ such that $\mathbf{r}=\left(\mathbf{r}_{0}, \mathbf{r}_{1}, \mathbf{r}_{2}, \mathbf{r}_{3}\right)$ is a central configuration.

(a) Then, there exist unique mass ratios: 


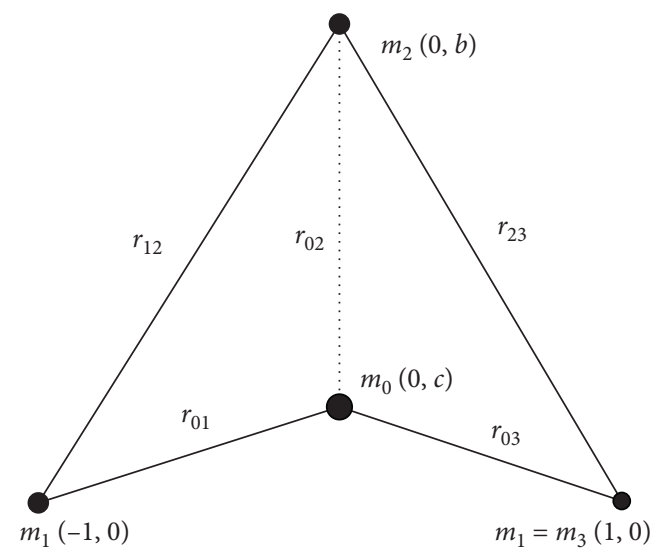

Figure 5: Concave four-body configurations with a pair of equal masses $\left(m_{1}=m_{3}\right)$.

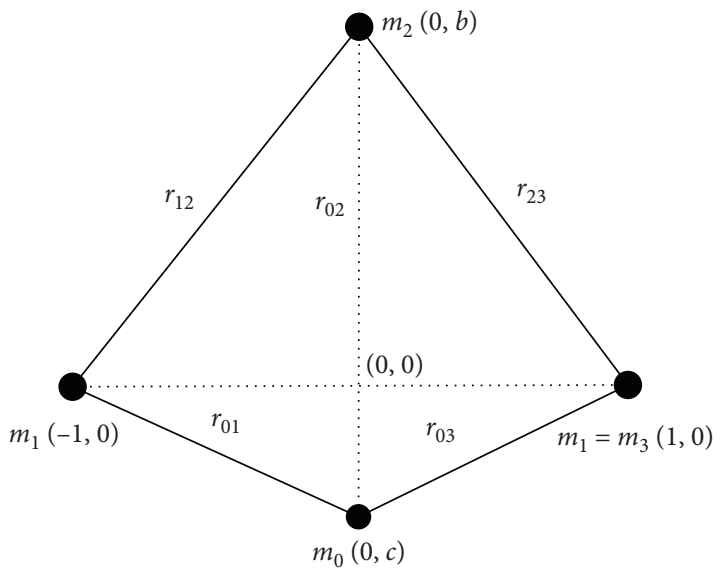

Figure 6: Convex four-body kite configurations with two equal masses $\left(m_{1}=m_{3}\right)$.

$$
\begin{aligned}
& \mu_{0}=\frac{m_{0}}{m_{1}}=\frac{b\left(8-\left(b^{2}+1\right)^{3 / 2}\right)(b-c)^{2}\left(c^{2}+1\right)^{3 / 2}}{4\left((b-c)^{3}-\left(c^{2}+1\right)^{3 / 2}\right)\left(b^{2}+1\right)^{3 / 2}}, \\
& \mu_{2}=\frac{m_{2}}{m_{1}}=\frac{c\left(8-\left(c^{2}+1\right)^{3 / 2}\right)(c-b)^{2}\left(b^{2}+1\right)^{3 / 2}}{4\left(\left(b^{2}+1\right)^{3 / 2}-(b-c)^{3}\right)\left(c^{2}+1\right)^{3 / 2}} .
\end{aligned}
$$

(b) The region of existence of central configuration where the four positive masses are arranged in a concave kite configuration is given by

$$
\begin{aligned}
R_{t}= & \left\{(b, c) \mid\left(0<c<\frac{1}{\sqrt{3}} \wedge c+\sqrt{c^{2}+1}<b<\sqrt{3}\right)\right. \\
& \left.\vee\left(\frac{1}{\sqrt{3}}<c<\sqrt{3} \wedge \sqrt{3}<b<c+\sqrt{c^{2}+1}\right)\right\} .
\end{aligned}
$$

(c) The region of existence of central configuration where the four positive masses are arranged in a convex kite configuration is given by

$$
\begin{aligned}
R_{r}= & \left\{(b, c) \mid\left(-\sqrt{3}<c \leq-\frac{1}{\sqrt{3}} \wedge c+\sqrt{c^{2}+1}<b<\sqrt{3}\right)\right. \\
& \left.\vee\left(-\frac{1}{\sqrt{3}}<c<-2+\sqrt{3} \wedge \frac{c^{2}-1}{2 c}<b<\sqrt{3}\right)\right\} .
\end{aligned}
$$

Proof of Theorem 3. Consider four point masses with position vectors $\left(\mathbf{r}_{0}, \mathbf{r}_{1}, \mathbf{r}_{2}, \mathbf{r}_{3}\right)$ and masses $m_{0}, m_{1}, m_{2}$, and $m_{3}$, where $m_{3}=m_{1}$. The solution of $f_{01}=0$ and $f_{12}=0$ gives

$$
\begin{aligned}
& \mu_{0}=\frac{m_{0}}{m_{1}}=\frac{\left(R_{12}-R_{13}\right) \Delta_{123}}{\left(R_{01}-R_{02}\right) \Delta_{012}}=\frac{\beta \gamma b(\alpha-8)}{4 \alpha(b-c)(\beta-\gamma)}, \\
& \mu_{2}=\frac{m_{2}}{m_{1}}=\frac{\left(R_{01}-R_{13}\right) \Delta_{013}}{\left(R_{12}-R_{02}\right) \Delta_{012}}=\frac{\alpha \gamma c(8-\beta)}{4 \beta(b-c)(\alpha-\gamma)},
\end{aligned}
$$

where $\alpha=\left(1+b^{2}\right)^{3 / 2}, \beta=\left(1+c^{2}\right)^{3 / 2}$, and $\gamma=(b-c)^{3}$. The mass ratios $\mu_{0}$ and $\mu_{2}$ are well-defined functions of $b$ and $c$ except at $b=c$ and $b=c \pm \sqrt{1+c^{2}}$.

To find central configuration regions where $\mu_{0}>0$, it is sufficient to show that $\alpha-8$ and $\beta-\gamma$ have the same sign. It is trivial to see that $R \alpha-8=0$ when $b=\sqrt{3}$. Similarly, $\beta-$ $\gamma=0$ when $b=c+\sqrt{c^{2}+1}$. Hence, $\beta-\gamma$ is positive in

$$
R_{a}=\left\{(a, b) \mid b>0 \wedge c<b \wedge b<c+\sqrt{c^{2}+1}\right\} .
$$

The complement of $R_{a}$, where $\beta-\gamma<0$, is given by

$$
R_{a}^{c}=\left\{(a, b) \mid b>0 \wedge c<\left(b^{2}-1\right)(2 b)^{-1}\right\} .
$$

It is to be noted that the sign of $c$ will be determined according to whether the four-body configuration is concave $(c>0)$ or convex $(c<0)$. After some simplifications, the central configuration region for $\mu_{0}>0, c>0$ is given by

$$
\begin{aligned}
R_{t_{1}}= & \left\{(b, c) \mid\left(0<c<\frac{1}{\sqrt{3}} \wedge \sqrt{c^{2}+1}+c<b<\sqrt{3}\right)\right. \\
& \vee\left(\frac{1}{\sqrt{3}}<c \leq \sqrt{3} \wedge \sqrt{3}<b<\sqrt{c^{2}+1}+c\right) \\
& \left.\vee\left(c>\sqrt{3} \wedge c<b<\sqrt{c^{2}+1}+c\right)\right\} .
\end{aligned}
$$

Similarly, the central configuration region for $\mu_{0}>0, c<0$ is given by

$$
R_{r_{1}}=\left\{(b, c) \mid c<0, c+\sqrt{c^{2}+1}<b<\sqrt{3}\right\} .
$$

Consider the mass ratio $\mu_{2}$. Let $c>0$. Since $b>c$, therefore for $\mu_{2}>0,(\beta-8)(\alpha-\gamma)^{-1}$ must have the same sign. It is trivial to see that $8-\beta>0$ when $c \in(0, \sqrt{3})$ and $\alpha-\gamma>0$ for all $b>0$ and $c>0$. Therefore, $\mu_{2}>0$ in

$$
R_{t_{2}}=\{(b, c) \mid(0<b \leq \sqrt{3} \wedge 0<c<b) \vee(b>\sqrt{3} \wedge 0<c<\sqrt{3})\} \text {. }
$$

Similarly, when $c<0$ (rhomboidal configuration), the central configuration region where $\mu_{2}>0$ is given by 


$$
R_{r_{2}}=\{(b, c) \mid(0<b \leq \sqrt{3} \wedge 0<c<b) \vee(b>\sqrt{3} \wedge 0<c<\sqrt{3})\} .
$$

Hence, the region of existence of central configuration for the concave kite four-body problem where all the masses $m_{0}, m_{1}=m_{3}$, and $m_{2}$ are positive is given by $R_{t}=R_{t_{1}} \cap R_{t_{2}}$ and the corresponding convex kite central configuration region is given by $R_{r}=R_{r_{1}} \cap R_{r_{2}}$, where

$$
\begin{aligned}
& R_{t}=\left\{(b, c) \mid\left(0<c<(\sqrt{3})^{-1} \wedge c+\sqrt{c^{2}+1}<b<\sqrt{3}\right) \vee\left((\sqrt{3})^{-1}<c<\sqrt{3} \wedge \sqrt{3}<b<c+\sqrt{c^{2}+1}\right)\right\}, \\
& R_{r}=\left\{(b, c) \mid\left(-\sqrt{3}<c \leq-(\sqrt{3})^{-1} \wedge c+\sqrt{c^{2}+1}<b<\sqrt{3}\right) \vee\left(-(\sqrt{3})^{-1}<c<\sqrt{3}-2 \wedge\left(c^{2}-1\right)(2 c)^{-1}<b<\sqrt{3}\right)\right\} .
\end{aligned}
$$

The regions $R_{t}$ and $R_{r}$ are shown in Figures 7 and 8, respectively.

4.2. Action Minimizing Orbits in the Convex Kite Four-Body Problem. In this section, we discuss the minimization property of a four-body problem which has two equal masses $\left(m_{1}=m_{3}\right)$ on the horizontal axis and two positive masses $m_{2}$ and $m_{0}$ on the vertical axis, which is also the axis of symmetry.

Theorem 4. For the four-body problem considered in Theorem 1, the minimizers for $A(q)$ restricted to the homographic solutions $q_{i}(t)=\phi(t) q_{i, 0}$ are precisely the Keplerian elliptical solutions, and the minimum of the action is equal to $(3 / 2)(2 \pi)^{2 / 3} T^{1 / 3}(\xi(b, c) / \eta(b, c))^{2 / 3}$.

Let us call $\Omega_{c m}$ the $y$-coordinate of the center of mass in the configuration described earlier in Section 3.1, then

$$
\Omega_{c m}=\frac{m_{0} c+m_{2} b}{m_{0}+2 m_{1}+m_{2}}
$$

and the center of mass is $\mathrm{COM}=\left(0, \Omega_{\mathrm{cm}}\right)$. Observe that

$$
\begin{aligned}
& r_{0}=\left|q_{0,0}\right|=\left|c-\Omega_{c m}\right|, \\
& r_{1}=r_{3}=\sqrt{1+\Omega_{c m}^{2}}, \\
& r_{2}=\left|b-\Omega_{c m}\right| .
\end{aligned}
$$

Proof of Theorem 4. The kinetic energy term $K$ is equal to

$$
\begin{aligned}
K & =\frac{1}{2} \sum_{i=0}^{3} m_{i}\left|\dot{q}_{i}(t)\right|^{2}=|\dot{\phi}(t)|^{2}\left(m_{1} r_{1}^{2}+\frac{m_{2}}{2} r_{2}^{2}+\frac{m_{0}}{2} r_{0}^{2}\right) \\
& =|\dot{\phi}(t)|^{2} r_{1}^{2}\left(m_{1}+\frac{1}{2}\left(\frac{m_{2} r_{2}^{2}+m_{0} r_{0}^{2}}{r_{1}^{2}}\right)\right) \\
& =\left|\dot{q}_{1}(t)\right|^{2}\left(m_{1}+\frac{1}{2}\left(\frac{m_{2} r_{2}^{2}+m_{0} r_{0}^{2}}{r_{1}^{2}}\right)\right),
\end{aligned}
$$

where we have used the fact that $\left|\dot{q}_{1}(t)\right|^{2}=|\dot{\phi}(t)|^{2} r_{1}^{2}$.

On the other hand, the potential energy is given by

$$
U=\sum_{0 \leq i<j \leq 3} \frac{m_{i} m_{j}}{\left|q_{i}-q_{j}\right|},
$$

and using $\left|q_{i}-q_{j}\right|=|\phi(t)|\left|q_{i, 0}-q_{j, 0}\right|$, we get

$$
U=\frac{1}{|\phi(t)|}\left(\frac{m_{1}^{2}}{2}+\frac{2 m_{1} m_{2}}{\sqrt{1+b^{2}}}+\frac{2 m_{1} m_{0}}{\sqrt{1+c^{2}}}+\frac{m_{2} m_{0}}{|c-b|}\right) \text {. }
$$

Multiplying and dividing by $r_{1}$, we obtain

$$
U=\frac{1}{\left|q_{1}\right|}\left(\frac{m_{1}^{2} r_{1}}{2}+\frac{2 m_{1} m_{2} r_{1}}{\sqrt{1+b^{2}}}+\frac{2 m_{1} m_{0} r_{1}}{\sqrt{1+c^{2}}}+\frac{m_{2} m_{0} r_{1}}{|c-b|}\right) .
$$

Defining $\mu_{0}=m_{0} / m_{1}, \mu_{2}=m_{2} / m_{1}$, and $m_{1}=1$,

$$
U=\frac{1}{\left|q_{1}\right|}\left(\frac{r_{1}}{2}+\frac{2 \mu_{2} r_{1}}{\sqrt{1+b^{2}}}+\frac{2 \mu_{0} r_{1}}{\sqrt{1+c^{2}}}+\frac{\mu_{2} \mu_{0} r_{1}}{|c-b|}\right) .
$$

Now, we are ready to compute the action restricted to this class of homographic solutions:

$$
\begin{aligned}
A= & \int_{0}^{T}\left(1+\frac{1}{2}\left(\frac{\mu_{2} r_{2}^{2}+\mu_{0} r_{0}^{2}}{r_{1}^{2}}\right)\right) \frac{\left|\dot{q}_{1}\right|^{2}}{2} \mathrm{~d} t \\
& +\int_{0}^{T}\left(\frac{r_{1}}{2}+\frac{2 \mu_{2} r_{1}}{\sqrt{1+b^{2}}}+\frac{2 \mu_{0} r_{1}}{\sqrt{1+c^{2}}}+\frac{\mu_{2} \mu_{0} r_{1}}{|c-b|}\right) \frac{1}{\left|q_{1}\right|} \mathrm{d} t \\
= & 2+\left(\frac{\mu_{2} r_{2}^{2}+\mu_{0} r_{0}^{2}}{r_{1}^{2}}\right) \int_{0}^{T} \frac{\left|\dot{q}_{1}\right|^{2}}{2} \mathrm{~d} t \\
& +\left(\frac{r_{1}}{2}+\frac{2 \mu_{2} r_{1}}{\sqrt{1+b^{2}}}+\frac{2 \mu_{0} r_{1}}{\sqrt{1+c^{2}}}+\frac{\mu_{2} \mu_{0} r_{1}}{|c-b|}\right) \int_{0}^{T} \frac{1}{\left|q_{1}\right|} \mathrm{d} t .
\end{aligned}
$$

Let

$$
\begin{aligned}
& \eta(b, c)=1+\frac{1}{2}\left(\frac{\mu_{2} r_{2}^{2}+\mu_{0} r_{0}^{2}}{r_{1}^{2}}\right), \\
& \xi(b, c)=\frac{r_{1}}{2}+\frac{2 \mu_{2} r_{1}}{\sqrt{1+b^{2}}}+\frac{2 \mu_{0} r_{1}}{\sqrt{1+c^{2}}}+\frac{\mu_{2} \mu_{0} r_{1}}{|c-b|} .
\end{aligned}
$$

Therefore,

$$
A(q)=\eta \int_{0}^{T} \frac{\left|\dot{q}_{1}\right|^{2}}{2} \mathrm{~d} t+\xi \int_{0}^{T} \frac{1}{\left|q_{1}\right|} \mathrm{d} t .
$$

The infimum of $A(q)$ is 


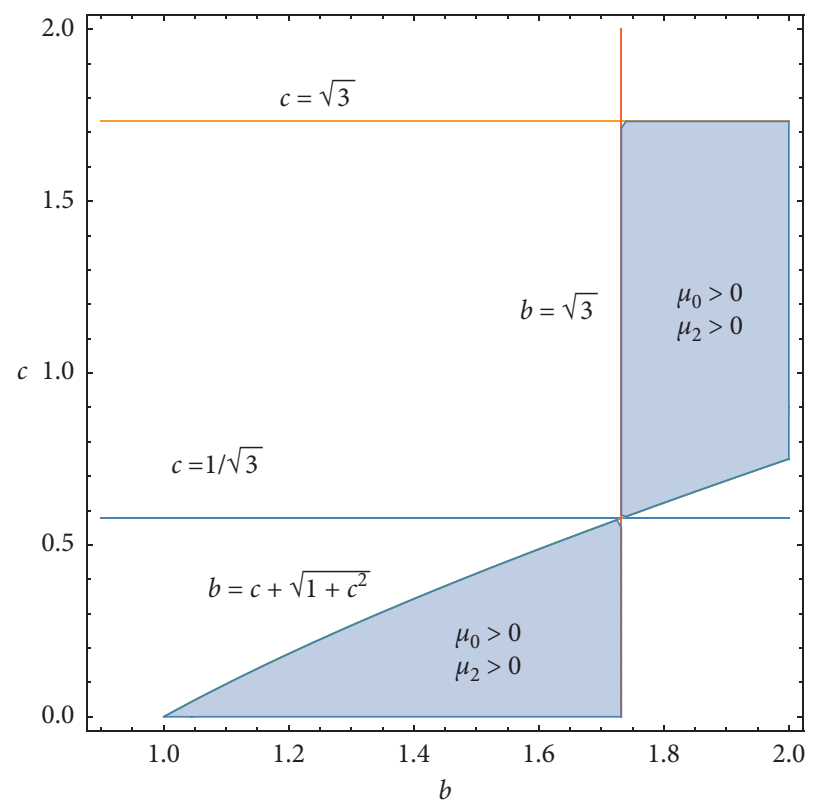

FIGURE 7: Region of existence of central configuration for the concave kite four-body configuration where $m_{1}=m_{3}=m \neq m_{0} \neq m_{2}$.

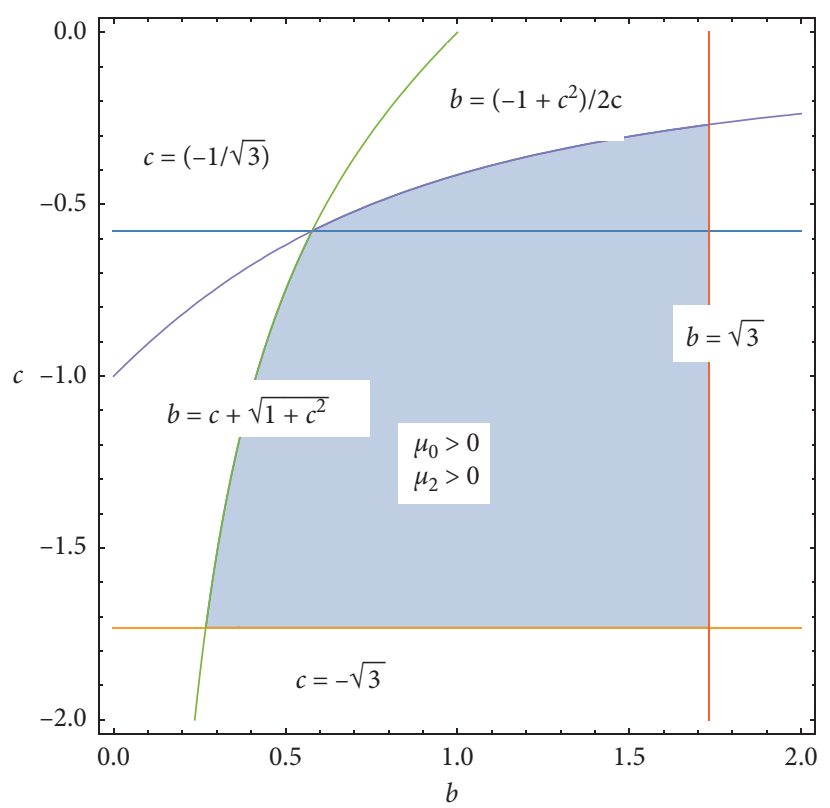

FIgURE 8: Region of existence of central configuration for the convex kite four-body configuration where $m_{1}=m_{3}=m \neq m_{0} \neq m_{2}$.

$$
\begin{aligned}
\inf _{q} A(q) & =\inf _{b>0, c>0} \inf _{q_{1}}\left\{\eta \int_{0}^{T} \frac{\left|\dot{q}_{1}\right|^{2}}{2} \mathrm{~d} t+\xi \int_{0}^{T} \frac{1}{\left|q_{1}\right|} \mathrm{d} t\right\} \\
& =\inf _{b>0, c>0}\left\{\eta \inf _{q_{1}}\left(\int_{0}^{T} \frac{\left|\dot{q}_{1}\right|^{2}}{2} \mathrm{~d} t+\frac{\xi}{\eta} \int_{0}^{T} \frac{1}{\left|q_{1}\right|} \mathrm{d} t\right)\right\} .
\end{aligned}
$$

By Gordon's result, we have

$$
\begin{gathered}
\inf _{q_{1}}\left(\int_{0}^{T} \frac{\left|\dot{q}_{1}\right|^{2}}{2} \mathrm{~d} t+\frac{\xi}{\eta} \int_{0}^{T} \frac{1}{\left|q_{1}\right|} \mathrm{d} t\right) \\
=\frac{3}{2}(2 \pi)^{2 / 3} T^{1 / 3}\left(\frac{\xi(b, c)}{\eta(b, c)}\right)^{2 / 3} .
\end{gathered}
$$



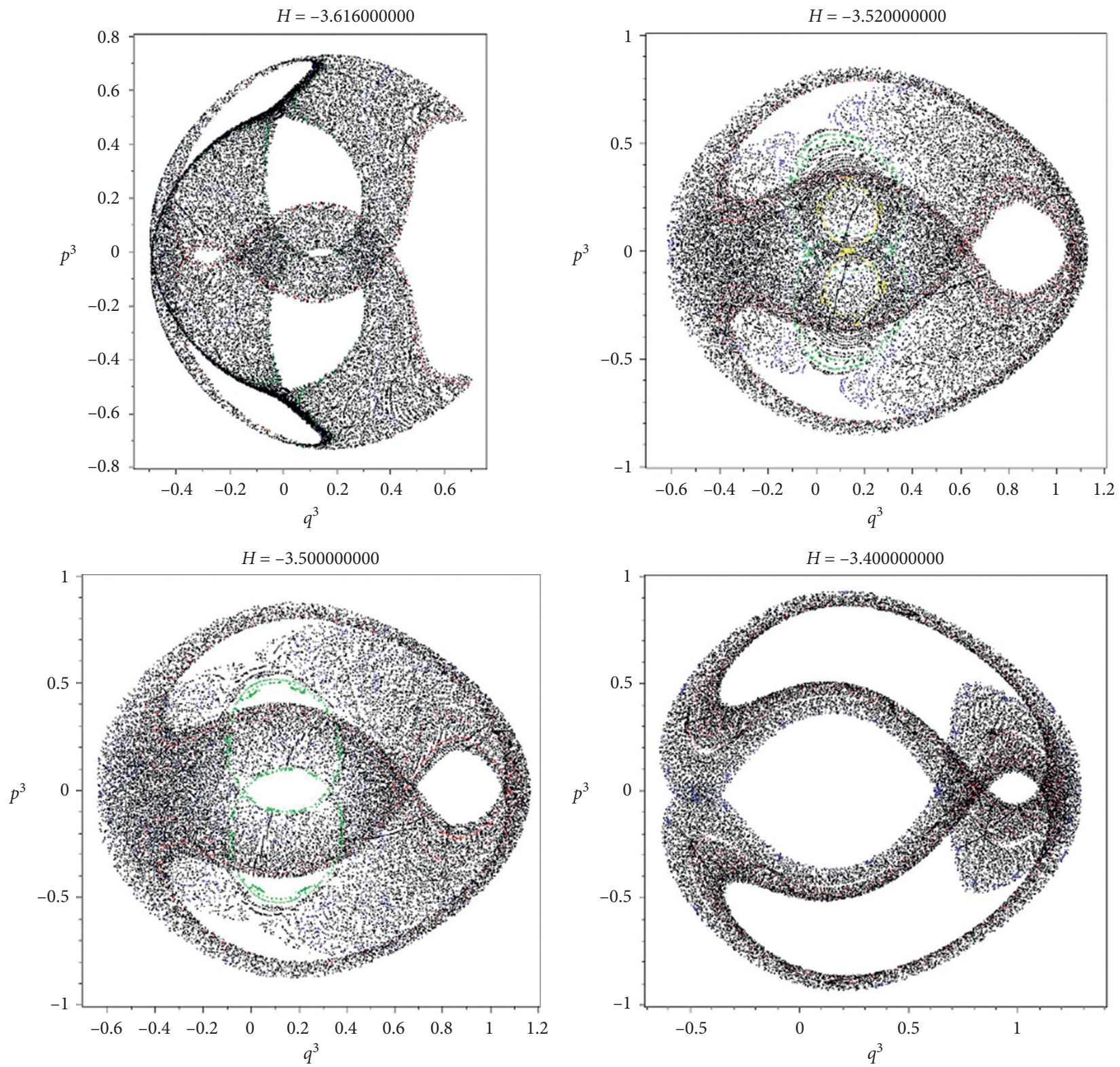

Figure 9: The progressive evolution of a Poincaré surface of sections for different energy levels in the unequal masses case $\left(m_{0}=1.28155\right.$, $m_{2}=0.31, m_{3}=m_{1}=1, b=1.1$, and $\left.c=0.1\right)$.

Then,

$$
\begin{aligned}
\inf _{q} A(q) & =\inf _{b>0, c>0}\left\{\eta(b, c) \frac{3}{2}(2 \pi)^{2 / 3} T^{1 / 3}\left(\frac{\xi(b, c)}{\eta(b, c)}\right)^{2 / 3}\right\} \\
& =\inf _{b>0, c>0}\left\{\frac{3}{2}(2 \pi)^{2 / 3} T^{1 / 3} \eta(b, c)^{1 / 3}(\xi(b, c))^{2 / 3}\right\} .
\end{aligned}
$$

Let $\phi(b, c)=(3 / 2)(2 \pi)^{2 / 3} T^{1 / 3} \eta(b, c)^{1 / 3}(\xi(b, c))^{2 / 3}$, and then $\phi(b, c)$ attains its infimum at $\left(b_{0}, c_{0}\right)$ if and only if $\eta(b, c)(\xi(b, c))^{2}$ attains its infimum at $\left(b_{0}, c_{0}\right)$. Similar to the concave case, we need to show that for positive values of $b_{0}$ and $c_{0}$, the function $\phi(b, c)$ is convex and coercive. For convexity, we compute the Hessian matrix for $\phi(b, c)$ and numerically show that $H(b, c)$ is positive semidefinite in the region $R_{r}$.
That concludes that the function $\phi(b, c)$ is convex. For coercivity, we see that $\phi(b, c)$ is continuous for all positive values of $b$ and $c, \phi(b, c) \longrightarrow \infty$ as $(b, c) \longrightarrow(0,0)$, and when $b \longrightarrow \infty$ and $c \longrightarrow \infty, \phi(b, c)$ tends to $\infty$, which implies $\phi(b, c)$ is coercive. Hence, $\phi(b, c)$ attains $\inf _{b>0, c>0}$ $\{\phi(b, c)\} \quad$ at unique $\left(b_{0}, c_{0}\right), \quad b_{0}>0, \quad c_{0}>0$, and satisfies $\phi\left(b_{0}, c_{0}\right)=0$.

\section{Hamiltonian Formulation of the Problem: Some Numerical Examples}

It is well known that the study of the trajectories of celestial bodies under their mutual gravitational attractions is important for understanding their movement and 

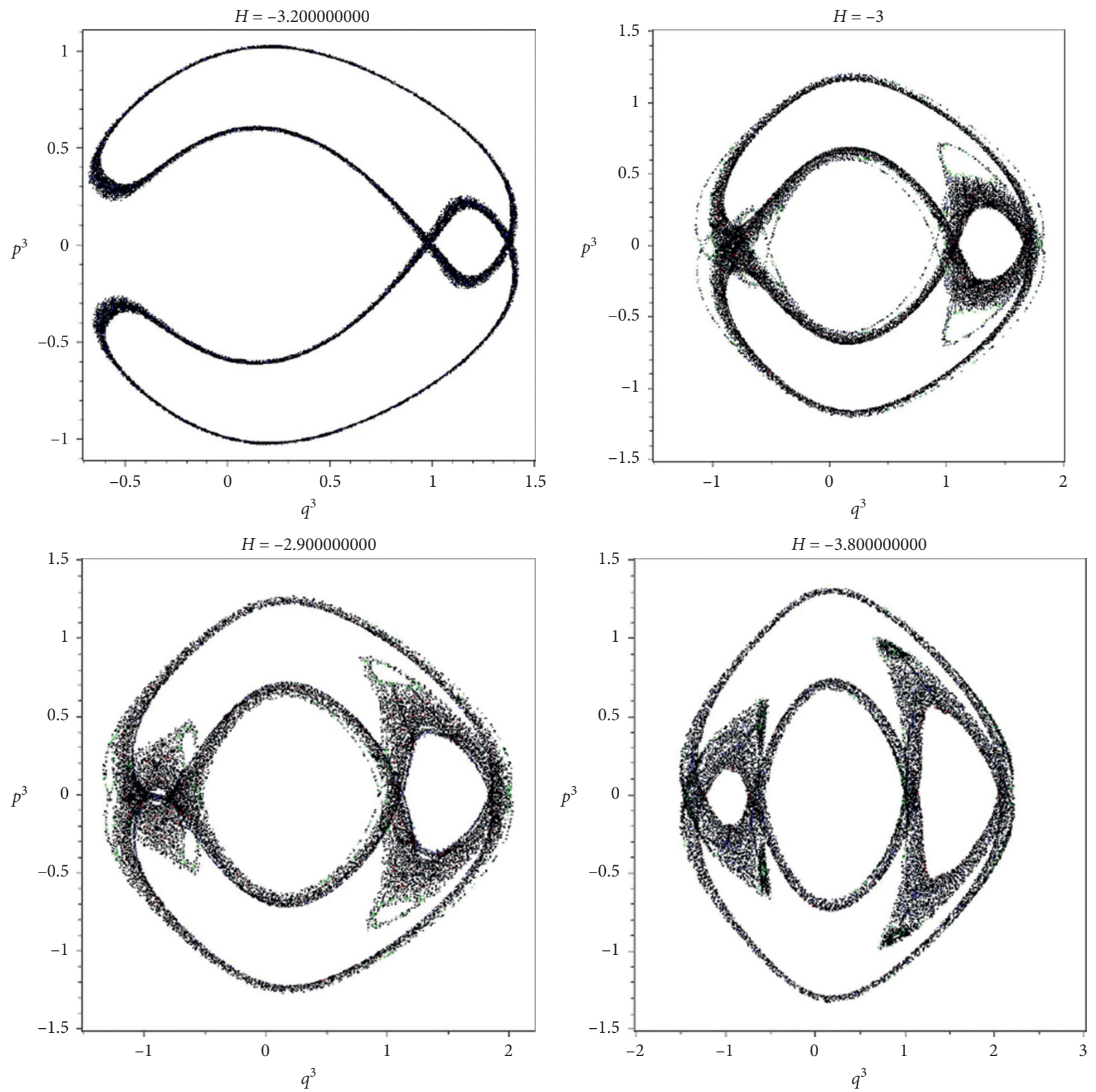

Figure 10: The progressive evolution of a Poincaré surface of sections for different energy levels in the unequal masses case $\left(m_{0}=1.28155\right.$, $m_{2}=0.31, m_{3}=m_{1}=1, b=1.1$, and $\left.c=0.1\right)$.

navigation. In that sense, a special type of the four-body problem with analytical and numerical investigation can contribute to the understanding of the dynamical behavior of quadruple stellar systems (e.g., the HD 98800 quadruple system with two pairs of stars orbiting each other). Several authors studied the stability and dynamical evolution of symmetric quadruple systems for stars and exoplanetary systems of two planets $[40,41]$. In this section, we will study the periodic behavior of the kite four-body problem.
Consider the four-body problem introduced in Lemma 1 , which has a pair of equal masses and one axis of symmetry. Using the symmetries and position coordinates from Lemma 1, we obtain the following reduced Hamiltonian in the case of unequal masses:

$$
H=\sum_{i=0}^{3} \frac{p_{i}^{2}}{2 m_{i}}-\frac{m_{0} m_{1}}{r_{01}}-\frac{m_{0} m_{2}}{r_{02}}-\frac{m_{0} m_{3}}{r_{03}}-\frac{m_{1} m_{2}}{r_{12}}-\frac{m_{1} m_{3}}{r_{13}}-\frac{m_{2} m_{3}}{r_{23}},
$$

where 

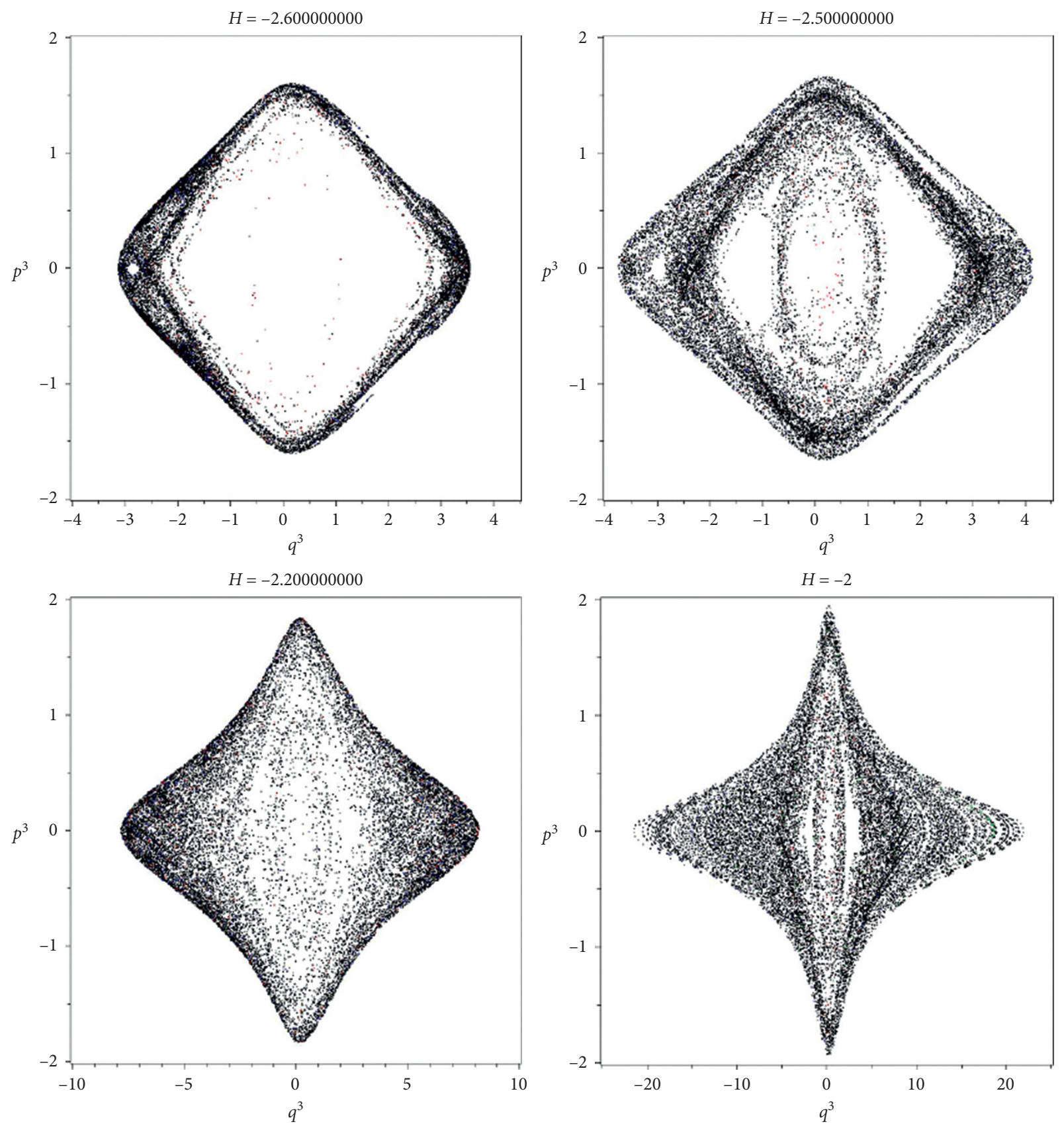

Figure 11: The progressive evolution of a Poincare surface of sections for different energy levels in the unequal masses case $\left(m_{0}=1.28155\right.$, $m_{2}=0.31, m_{3}=m_{1}=1, b=1.1$, and $\left.c=0.1\right)$.

$$
\begin{aligned}
r_{01}^{2} & =1+\left(q_{1}-c\right)^{2}, \\
r_{02}^{2} & =(b-c)^{2}, \\
r_{03}^{2} & =1+\left(q_{3}-c\right)^{2}, \\
r_{12}^{2} & =1+\left(q_{1}-b\right)^{2}, \\
r_{13}^{2} & =4+\left(q_{1}-q_{3}\right)^{2}, \\
r_{23}^{2} & =1+\left(b-q_{3}\right)^{2}, \\
b & >c,
\end{aligned}
$$

and $q_{i}$ and $p_{i}, i=\overline{0,3}$, are the generalized coordinates and momenta (we assume for simplicity that the gravitational constant is equal to 1 ).

For the investigation of the reduced Hamiltonian equations of motion (75), we have selected examples from concave and convex kite four-body problems introduced in Section 2. We have used classical numerical methods and found periodic orbits for a given vector field. The stability of a periodic orbit for an autonomous vector field can be calculated by Poincaré maps, which replaces the flow of the $n$-dimensional continuous vector field with an 

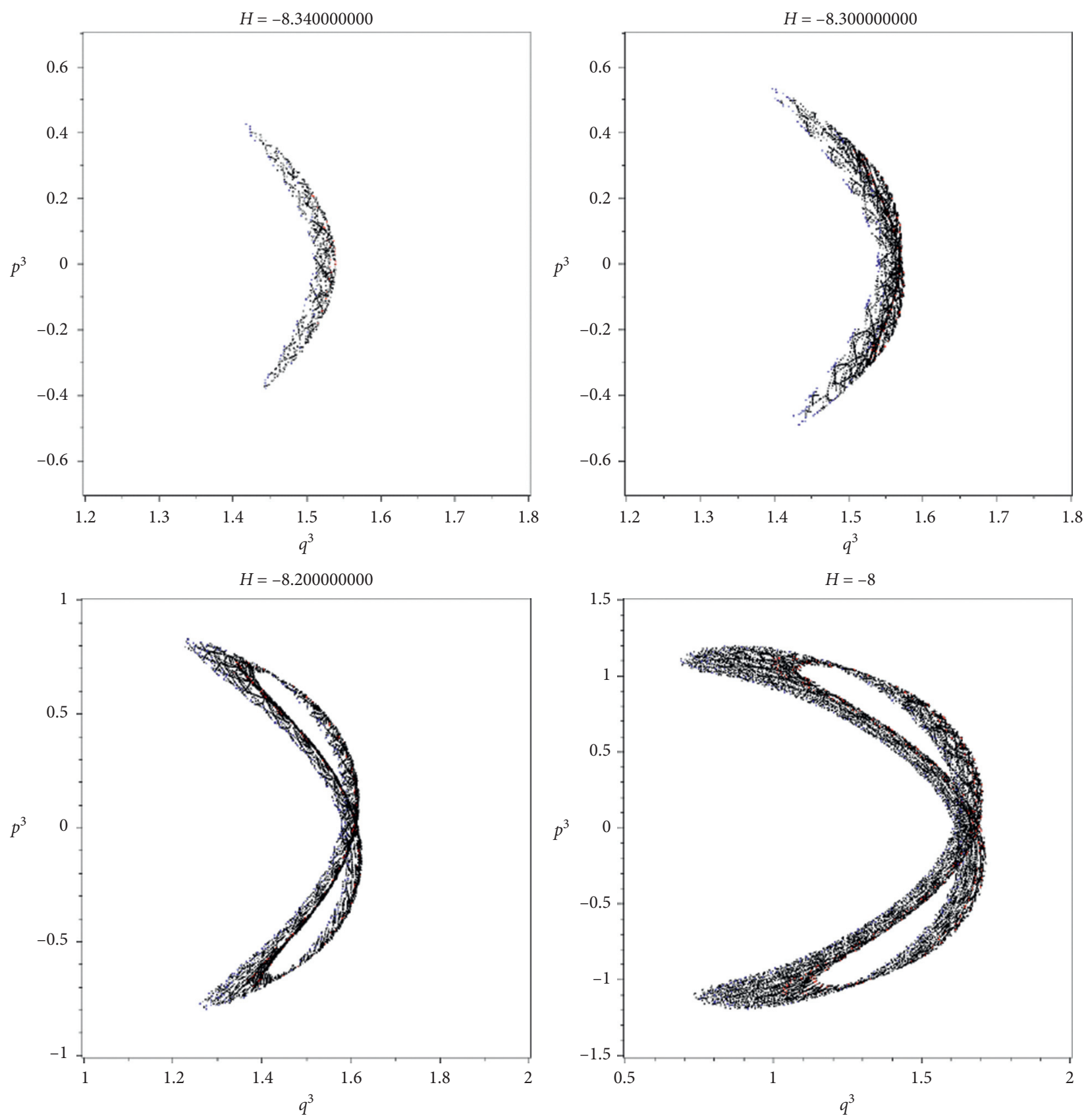

FIgURE 12: The progressive changing of a Poincaré surface of sections for different energy levels in the unequal masses case $\left(m_{0}=2.658\right.$, $m_{2}=1.03, m_{3}=m_{1}=1, b=1.9$, and $\left.c=0.7\right)$.

$(n-1)$-dimensional map [42]. In this article, the analysis of periodic and quasiperiodic orbits is performed using the Poincaré surface of the section technique by picking the phase element $p_{1}=0$. In the $n$-dimensional case, the Poincaré surface of the section has dimensionality $2 n-2$. The intersection points of the solution curves with the corresponding $\left(p_{i}, q_{i}\right), i=\overline{0, n-1}$, plane still lie on a smooth curve [43].

In order to construct the Poincaré surface of the section and to find the corresponding periodic orbits, we plot the motion from the $4 \mathrm{D}$ phase space $\left(q_{1}, q_{3}, p_{1}, p_{3}\right)$ in a "cut plane" $p_{1}(t)=0, q_{1} t>0$. Since $H$ is conserved, any point on this surface of the section will uniquely define the orbit. Our Poincaré surface of sections (Figures 9-11) describe the concave four-body problem in the unequal mass case with energy values $E \in\{-3.52, \ldots,-2\}$, plotting $q_{3}(t)$ versus $p_{3}(t)$, and $m_{0}=1.28155, m_{2}=0.31$, $m_{3}=m_{1}=1, b=1.1$, and $c=0.1$. The initial conditions considered here satisfy the central configuration equations introduced in Section 2. We wish to note that in case of the energy level -3.52 , we can see many little islands of quasiperiodic orbits. It should be emphasized that increasing the energy levels increase the Poincaré surface of sections in size, as a blowing up effect (see Figures 9-11). 

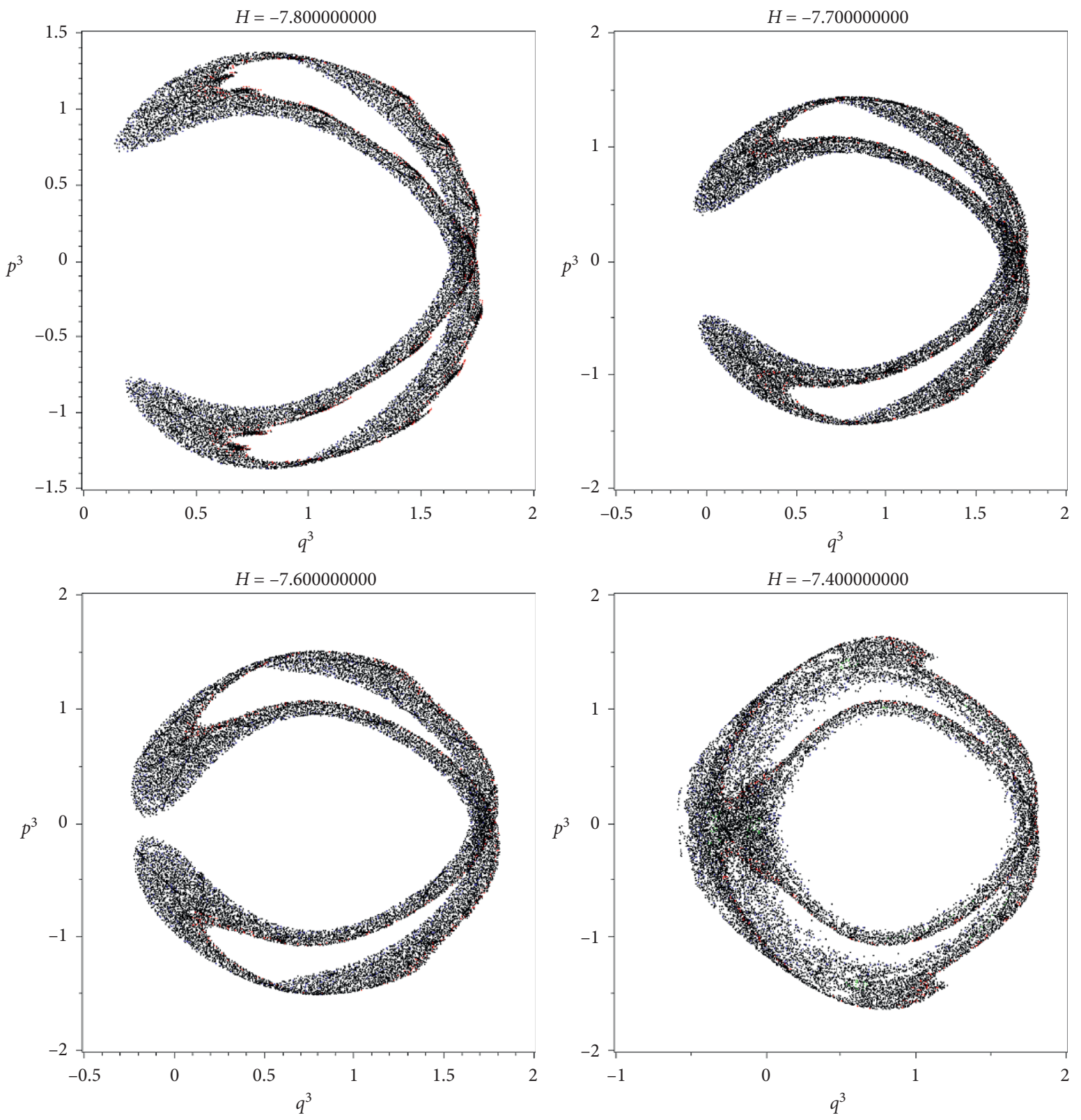

Figure 13: The progressive changing of a Poincaré surface of sections for different energy levels in the unequal masses case $\left(m_{0}=2.658\right.$, $m_{2}=1.03, m_{3}=m_{1}=1, b=1.9$, and $\left.c=0.7\right)$.

Another interesting unequal mass case is for increased masses $m_{0}=2.658, m_{2}=1.03$, and $m_{3}=m_{1}=1$ compared to the previous case. Similarly, using different energy levels, in this case for energy values $E \in\{-8.34, \ldots,-7.3\}$, the variation of the "horseshoe with open side to the left" as the letter $D$ shape to the "horseshoe with open side to the right" as the letter C shape can be observed (Figures 12-14). As earlier, in this particular case, we observe quasiperiodic orbits and the increasing size of the Poincare surface of sections.

In the equal mass case, we have chosen the following particular case: $m_{4}=5.17662, m=1, b=1.9$, and $c=0.7$. In this situation, we detected an interesting transformation of the Poincaré surface of sections through the energy levels $E \in\{-13.6, \ldots,-11\}$. In the case of lower energy levels, the outside part of the orbit indicates chaotic behavior. Moreover, by increasing the energy levels, the inside part of the orbit disintegrates, but the size of the Poincaré surface of sections has just a minor variation (Figure 15).

Consequently, we developed the surface of sections with around 300 points, which presents the progressive disintegration of the surfaces of solution curves of regular motion (KAM surface) generated with the increase in $H$ from -3.615 to -2 in our first example (Figures 9-11), from -8.34 to -7 in our second example (Figures 12-14), 

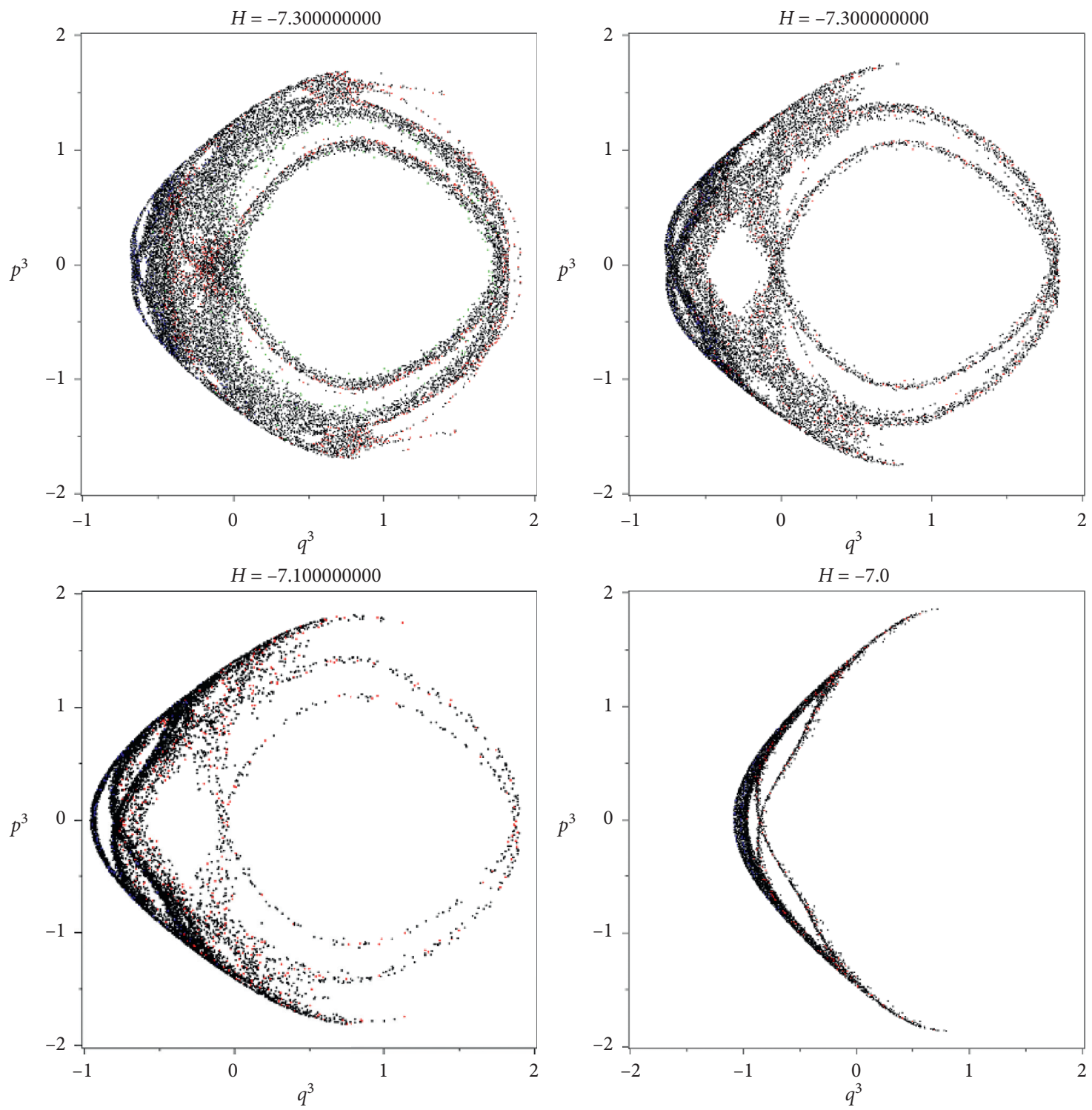

Figure 14: The progressive changing of a Poincaré surface of sections for different energy levels in the unequal masses case $\left(m_{0}=2.658\right.$, $m_{2}=1.03, m_{3}=m_{1}=1, b=1.9$, and $\left.c=0.7\right)$.

and from -13.6 to -11 in our third example (Figure 15). It seems that there exist invariant curves (for example, in the plots for $H$ equal to -3.5$)$ near resonances. These invariant curves could form "island sequences" (small groups in a row) and "islands cycles" (small islands inside of islands). One can observe that transitions between levels fuse at progressive integration, and the KAM surfaces progressively disappear.

In both cases (nonequal and equal masses), the surfaces show unique types of orbits, including quasiperiodic and island orbits. Let us mention that comparing both cases, when the values of $m_{0}$ are increased, then there is a noticeable effect influence on the stability and the existence of quasiperiodic orbits in concave and convex kite four-body problems. In other words, we conjecture that the increasing central mass plays a stabilizing role.

The described Poincaré surface of sections allows to study the local stability of the kite four-body problem, the transition from ordered to stochastic motion. They contribute significantly in numerical studies and in verification of the concordance between analytical and numerical results.

\section{Conclusions}

In this paper, we investigated the central configurations of convex and concave kite four-body problems, deriving 

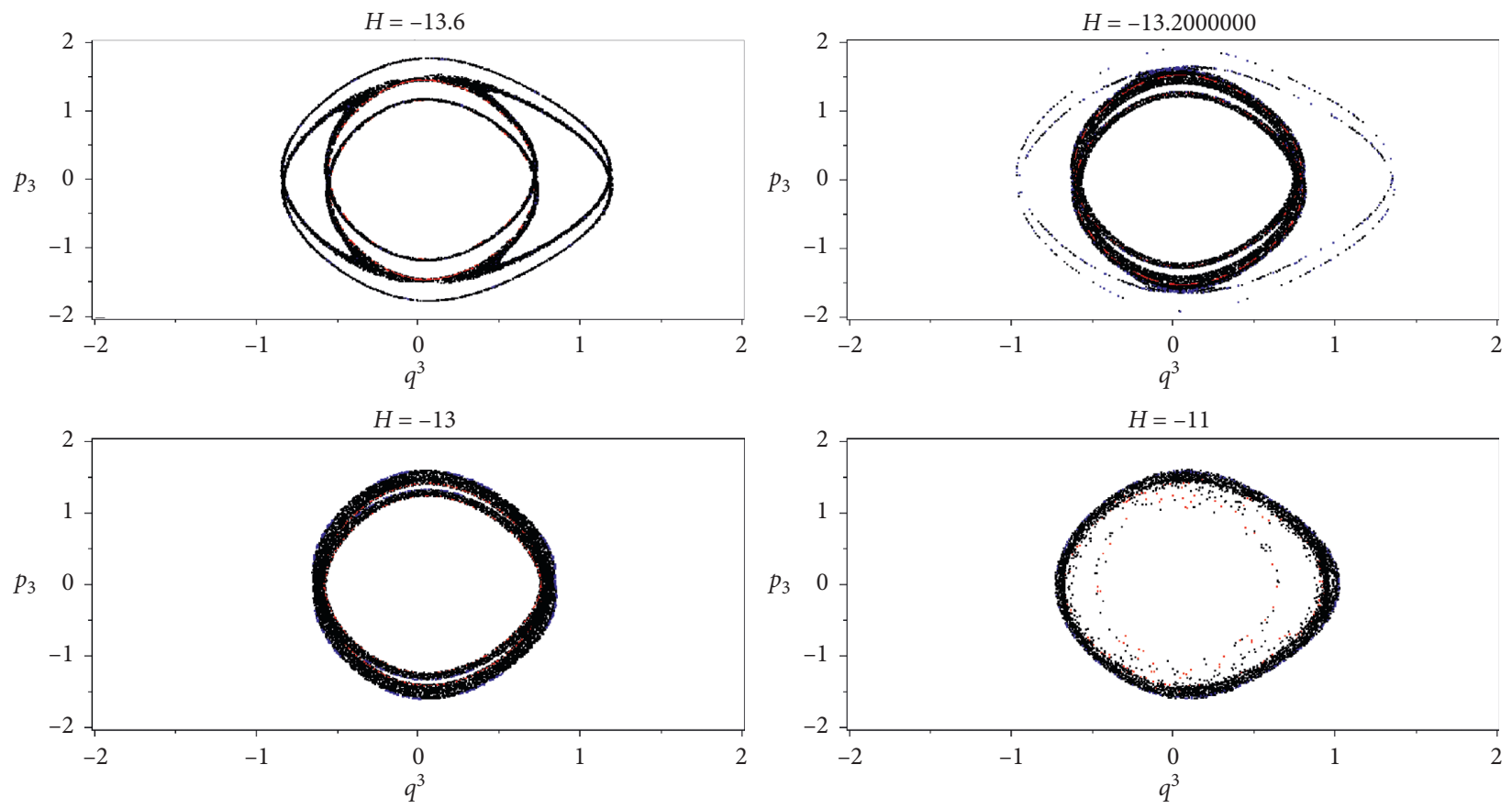

FiguRE 15: The progressive transformation of a Poincaré surface of sections for different energy levels in the equal masses case $\left(m_{0}=5.17662, m=1, b=1.9\right.$, and $\left.c=0.7\right)$.

regions of central configuration. Three four-body arrangements are discussed which include two concave four-body configurations and one convex four-body configuration. In one of the concave configurations, three of the masses on the vertices of the triangle are equal and the mass on the axis of symmetry can take various positive values. In the second case of concave configuration and the case of the convex configuration, there is only a pair of equal masses and the two masses on the axis of symmetry are nonequal. In each of the three cases, regions of central configuration are derived for positive masses. In the first concave case, we can write the mass ratios as a function of one variable and show its optimum values give values of the parameter. In the other two cases, the mass ratios are written as functions of two variables. The action minimizing orbits for both the concave and convex configurations is analyzed, and it is shown that the minimizers of the action functional restricted to the homographic solutions are the Keplerian elliptical solutions. Using the Hamiltonian formalism, we have identified regions with periodic and quasiperiodic orbits. Moreover, we studied the chaotic behavior in the phase space utilizing the Poincaré surface of sections. It was shown that increasing the value of the central mass $m_{0}$ plays a stabilizing role in the case of both convex and concave four-body problems.

\section{Data Availability}

No data were used to support this study.

\section{Conflicts of Interest}

The authors declare that they have no conflicts of interest.

\section{Acknowledgments}

I. Szücs-Csillik was partially supported by a grant of the Romanian Ministry of National Education and Scientific Research, RDI Programme for Space Technology and Advanced Research-SAFE SPACE, project number 236/ 04.04.2018. II. Daniel Offin was partially supported by a Discovery Grant from NSERC of Canada.

\section{References}

[1] C. Deng and S. Zhang, "Planar symmetric concave central configurations in Newtonian four-body problems," Journal of Geometry and Physics, vol. 83, pp. 43-52, 2014.

[2] W. D. MacMillan and W. Bartky, "Permanent configurations in the problem of four bodies," Transactions of the American Mathematical Society, vol. 34, no. 4, p. 838, 1932.

[3] M. Shoaib, A. R. Kashif, and A. Sivasankaran, "Planar Central Configurations of symmetric five-body problems with two pairs of equal masses," Advances in Astronomy, vol. 2016, Article ID 9897681, 11 pages, 2016.

[4] C. Simó, "Relative equilibrium solutions in the four body problem," Celestial Mechanics, vol. 18, pp. 165-184, 1978.

[5] J. Llibre, "A note on the Dziobek central configurations," Proceedings of the American Mathematical Society, vol. 143, no. 8, pp. 3587-3591, 2015.

[6] J. Llibre, R. Moeckel, and C. Simó, Central Configurations, Springer, Basel, Switzerland, 2015.

[7] J. M. Cors and G. E. Roberts, "Four-body co-circular central configurations," Nonlinearity, vol. 25, no. 2, pp. 343-370, 2012.

[8] A. Albouy, Y. Fu, and S. Sun, "Symmetry of planar four-body convex central configurations," Proceedings of the Royal Society of London A: Mathematical, Physical and Engineering Sciences, vol. 464, no. 2093, pp. 1355-1365, 2008. 
[9] M. Shoaib, A. R. Kashif, and I. Szücs-Csillik, "On the planar central configurations of rhomboidal and triangular four- and five-body problems," Astrophysics and Space Science, vol. 362, no. 10, p. 182, 2017.

[10] B. Érdi and Z. Czirják, "Central configurations of four bodies with an axis of symmetry," Celestial Mechanics and Dynamical Astronomy, vol. 125, no. 1, pp. 33-70, 2016.

[11] S. Smale, "Mathematical problems for the next century," The Mathematical Intelligencer, vol. 20, no. 2, pp. 7-15, 1998.

[12] V. Barutello and S. Terracini, "Action minimizing orbits in then-body problem with simple choreography constraint," Nonlinearity, vol. 17, no. 6, pp. 2015-2039, 2004.

[13] E. Perez-Chavela and M. Santoprete, "Convex four-body central configurations with some equal masses," Archive for Rational Mechanics and Analysis, vol. 185, no. 3, pp. 481-494, 2007.

[14] K.-C. Chen, "Action-minimizing orbits in the parallelogram four-body problem with equal masses," Archive for Rational Mechanics and Analysis, vol. 158, no. 4, pp. 293-318, 2001.

[15] K.-C. Chen, "Variational methods on periodic and quasiperiodic solutions for the N-body problem," Ergodic Theory and Dynamical Systems, vol. 23, no. 6, pp. 1691-1715, 2003.

[16] L. Bakker and S. Simmons, "Stability of the rhomboidal symmetric-mass orbit," Discrete \& Continuous Dynamical Systems-A, vol. 35, no. 1, pp. 1-23, 2015.

[17] J. Simmons and E. Perez-Chavela, "The rhomboidal four-body problem," Global Flow on the Total Collision manifold, The Geometry of Hamiltonian Systems:, Springer, New York, NY, USA, 1991.

[18] M. Gidea and J. Llibre, "Symmetric planar central configurations of five bodies: euler plus two," Celestial Mechanics and Dynamical Astronomy, vol. 106, no. 1, pp. 89-107, 2010.

[19] E. A. Lacomba and E. Pérez-Chavela, "A compact model for the planar rhomboidal 4-body problem," Celestial Mechanics and Dynamical Astronomy, vol. 54, no. 4, pp. 343-355, 1992.

[20] E. Piña and P. Lonngi, "Central configurations for the planar Newtonian four-body problem," Celestial Mechanics and Dynamical Astronomy, vol. 108, no. 1, pp. 73-93, 2010.

[21] M. Shoaib, B. A. Steves, and A. Széll, "Stability analysis of quintuple stellar and planetary systems using a symmetric five-body model," New Astronomy, vol. 13, no. 8, pp. 639-645, 2008.

[22] M. Shoaib, "Central configurations in the trapezoidal fourbody problems," Applied Mathematical Sciences, vol. 9, no. 40, pp. 1971-1979, 2015.

[23] A. Sivasankaran, B. A. Steves, and W. L. Sweatman, "A global regularisation for integrating the Caledonian symmetric fourbody problem," Celestial Mechanics and Dynamical Astronomy, vol. 107, no. 1-2, pp. 157-168, 2010.

[24] J.-h. Ji, X.-h. Liao, and L. Liu, "The phase space structure of the rhomboidal four-body problem," Chinese Astronomy and Astrophysics, vol. 24, no. 3, pp. 381-386, 2000.

[25] J. Waldvogel, "The rhomboidal symmetric four-body problem," Celestial Mechanics and Dynamical Astronomy, vol. 113, no. 1, pp. 113-123, 2012.

[26] D. Yan, "Existence and linear stability of the rhomboidal periodic orbit in the planar equal mass four-body problem," Journal of Mathematical Analysis and Applications, vol. 388, no. 2, pp. 942-951, 2012.

[27] L. F. Mello and A. C. Fernandes, "New classes of spatial central configurations for the -body problem," Nonlinear Analysis: Real World Applications, vol. 12, no. 1, pp. 723-730, 2011.
[28] W. B. Gordon, "A minimizing property of Keplerian orbits," American Journal of Mathematics, vol. 99, no. 5, pp. 961-971, 1977.

[29] S. Q. Zhang and Q. Zhou, "A minimizing property of Lagrangian solution," Acta Mathematica Sinica, English Series, vol. 17, no. 3, pp. 497-500, 2001.

[30] S. Zhang and Q. Zhou, "A minimizing property of Eulerian solutions," Celestial Mechanics and Dynamical Astronomy, vol. 90, no. 3-4, pp. 239-243, 2004.

[31] A. M. Mansur and D. C. Offin, "A minimizing property of homographic solutions," Acta Mathematica Sinica, English Series, vol. 30, no. 2, pp. 353-360, 2014.

[32] A. Mansur, D. Offin, and M. Lewis, "Instability for a family of homographic periodic solutions in the parallelogram four body problem," Qualitative Theory of Dynamical Systems, vol. 16, no. 3, pp. 671-688, 2017.

[33] A. Mansur, D. Offin, and A. Arsie, "Extensions to chen's minimizing equal mass parallelogram solutions," Taiwanese Journal of Mathematics, vol. 21, no. 6, pp. 1437-1453, 2017.

[34] M. Celli, "The central configurations of four masses $x,-x, y$, $-y$," Journal of Differential Equations, vol. 235, no. 2, pp. 668-682, 2007.

[35] M. Corbera and J. Llibre, "Central configurations of the 4body problem with masses $m_{1}=m_{2}>m_{3}=m_{4}=m>0$ and smmall," Applied Mathematics and Computation, vol. 246, pp. 121-147, 2014.

[36] Y. Deng, B. Li, and S. Zhang, "Some notes on four-body cocircular central configurations," Journal of Mathematical Analysis and Applications, vol. 453, no. 1, pp. 398-409, 2017.

[37] M. Corbera, J. M. Cors, and G. E. Roberts, “A four-body convex central configuration with perpendicular diagonals is necessarily a kite," Qualitative Theory of Dynamical Systems, vol. 17, no. 2, pp. 367-374, 2018.

[38] M. Corbera, J. M. Cors, J. Llibre, and E. Pérez-Chavela, "Trapezoid central configurations," Applied Mathematics and Computation, vol. 346, pp. 127-142, 2019.

[39] M. Santoprete, "Four-body central configurations with one pair of opposite sides parallel," Journal of Mathematical Analysis and Applications, vol. 464, no. 1, pp. 421-434, 2018.

[40] B. A. Steves and A. E. Roy, "Some special restricted four-body problems-I. Modelling the Caledonian problem," Planetary and Space Science, vol. 46, no. 11-12, pp. 1465-1474, 1998.

[41] A. E. Roy and B. A. Steves, "The caledonian symmetrical double binary four-body problem I: surfaces of zero-velocity using the energy integral," New Developments in the Dynamics of Planetary Systems, vol. 78, pp. 299-318, 2001.

[42] I. Szücs-Csillik, "The lie integrator and the hénon-heiles system," Romanian Astronomical Journal, vol. 20, no. 1, pp. 49-66, 2010.

[43] E. S. Cheb-Terrab and H. P. Oliveira, "Poincaré sections of Hamiltonian systems," Computer Physics Communications, vol. 95, no. 2-3, pp. 171-189, 1996. 